\title{
ARITHMETICAL PREDICATES AND FUNCTION QUANTIFIERS $\left({ }^{1}\right)$
}

\author{
BY \\ S. C. KLEENE
}

In a paper in these Transactions [5] entitled Recursive predicates and quantifiers we showed that (classically) the arithmetical predicates fall into a hierarchy according to the sequences of alternating number quantifiers by which they can be defined from recursive predicates. In the present paper we consider the extended class of number-theoretic predicates obtained by applying the two-sorted predicate calculus to recursive predicates of number variables and variables for one-place number-thoretic functions, and show that similarly these fall into a hierarchy according to the sequences of alternating function quantifiers by which they can be defined from arithmetical predicates.

We have obtained a similar hierarchy using quantification of function variables of each higher finite type. But for the hierarchy using numbertheoretic function variables which we give now the requisite theory of recursive functionals is already in print in [8], while for the hierarchy using function variables of higher finite types the principal new point is to develop such a theory. Hence we reserve the latter for a further paper which we plan to write under the title Analytic predicates and function quantifiers of higher finite types.

The predicates definable by using variables of all finite types, with natural number variables as the lowest type, have been studied by Tarski [23] and [24]. It is only a matter of detail that his variables of higher type are set variables and ours function variables. The result of this and our proposed paper on higher finite types that a larger class of number-theoretic predicates is obtained with each additional type is already given by Tarski's investigations (or for the first additional type, by Mostowski [16, 4.5] or Davis [2] or [3]). What is new (we believe) is the hierarchy within each such class, and the details concerning these hierarchies.

1. Recursive functions and predicates of function and number variables. 1.1 Hereafter we cite our Introduction to metamathematics [8] simply as "IM". Our notations and terminology not otherwise explained will be found there. The notations are indexed bottom p. 538 $\left({ }^{2}\right)$.

Received by the editors November 23, 1953 and, in revised form, July 24, 1954.

(1) Based in part on research supported by the John Simon Guggenheim Foundation and the Graduate School of the University of Wisconsin.

(2) The normal form and enumeration theorems for $l>0$ can be rewritten using $\bar{\alpha}$ instead of $\tilde{\alpha}$, where $\bar{\alpha}(x)=\prod_{i<x} p_{i}^{\alpha(i)+1}$ (cf. [7]). Thus in IM pp. 291, 292 we have $T_{1}^{\alpha}(z, a, x)$ $\equiv T_{1}^{1}(\widetilde{\alpha}(x), z, a, x)$. Putting $T_{1}^{1}(w, z, a) \equiv T_{1}^{1}\left(\prod_{i<\operatorname{lh}(w)} p_{i} \exp (w)_{i}-1, z, a, \operatorname{lh}(w)\right)$, then $T_{1}^{1}(\tilde{\alpha}(x), z, a, x) \equiv T_{1}^{1}(\bar{\alpha}(x), z, a)$. 
1.2 When $\alpha_{1}, \cdots, \alpha_{m}$ are unspecified one-place functions, and $\phi\left(a_{1}, \cdots, a_{n}\right)$ is general recursive uniformly in $\alpha_{1}, \cdots, \alpha_{m}$ (IM p. 275), we follow [7] in writing $\phi\left(a_{1}, \cdots, a_{n}\right)$ also as $\phi\left(\alpha_{1}, \cdots, \alpha_{m}, a_{1}, \cdots, a_{n}\right)$, and saying that the latter function of $m$ function and $n$ number variables is general recursive $\left.{ }^{3}\right)$.

More generally, if $\phi\left(a_{1}, \cdots, a_{n}\right)$ is general recursive in $\psi_{1}, \cdots, \psi_{l}$, $\alpha_{1}, \cdots, \alpha_{m}$ uniformly in $\alpha_{1}, \cdots, \alpha_{m}$, where $\alpha_{1}, \cdots, \alpha_{m}$ are as before and $\psi_{1}, \cdots, \psi_{l}$ are number-theoretic functions $\left.{ }^{4}\right)$ not necessarily one-place, we write $\phi\left(a_{1}, \cdots, a_{n}\right)$ also as $\phi\left(\alpha_{1}, \cdots, \alpha_{m}, a_{1}, \cdots, a_{n}\right)$ and call it then general recursive in $\psi_{1}, \cdots, \psi_{l}$.

These notions apply to primitive recursiveness similarly (cf. IM p. 234), and extend as usual via representing functions (IM p. 227) to the case $\phi$ or some or all of $\psi_{1}, \cdots, \psi_{l}$ are replaced by predicates.

Mostly we shall state our results for $l=0$, but up through $\S 5$ they will hold similarly for $l>0$.

1.3 We shall wish to perform substitutions for function variables as well as for number variables. This we can do, using the $\lambda$-notation of Church [1] (cf. IM p. 34).

For example, if $\phi(\beta, \alpha, a)$ and $\psi(\alpha, y, a)$ are general recursive functions of the indicated variables, then $\phi(\lambda y \psi(\alpha, y, a), \alpha, a)$ is a general recursive function of $\alpha$ and $a(5)$. This can be inferred either from IM Lemma VI p. 344 and the transitivity of relative recursiveness (IM Theorem II p. 275), or by use of the normal form theorem (IM Theorem IX* p. 292).

Via the representing function of $P$, if the predicate $P(\beta, \alpha, a)$ and function $\psi(\alpha, y, a)$ are general recursive, so is the predicate $P(\lambda y \psi(\alpha, y, a), \alpha, a)$.

More complicated substitutions can be handled similarly (cf. IM $\S 44)$.

2. Arithmetical and analytic predicates. $2.1 \mathrm{We}$ shall study predicates of our two types of variables number and function which are expressible explicitly in terms of given predicates and functions (the primitives) and the symbolism of the predicate calculus with the two types of variables; i.e. they are definable from the primitives by applying explicit definition, the propositional connectives $\rightarrow, \&, \vee$ and - , and the quantifiers $(E x),(x),(E \alpha)$ and $(\alpha)$ of the two kinds existential and generality and the two types number and function.

(3) In other words, now and in [7] we allow a general recursive functional $\mathbf{F}\left(\alpha_{1}, \cdots, \alpha_{m}\right.$; $\left.a_{1}, \cdots, a_{n}\right)$ in the notation of IM pp. 234, 275 to be written simply $\phi\left(\alpha_{1}, \cdots, \alpha_{m}, a_{1}, \cdots, a_{n}\right)$ and to be called simply a function, when $\alpha_{1}, \cdots, \alpha_{m}$ are all variables for one-place functions.

The restriction of our function variables to ones for one-place functions is for convenience; it saves us the necessity of stating for each the number of its arguments. We would get no wider class of predicates of the variables we allow by permitting quantification also of variables for several place functions.

$\left({ }^{4}\right)$ But not in this paper functions such as $\psi(\alpha, a)$, as the notion of relative recursiveness in $\mathrm{IM}$ is of a number-theoretic function in number-theoretic functions.

(5) The same is true reading "primitive recursive" in place of "general recursive", by the first method of proof with IM Lemma I p. 236 instead of Lemma VI. 
When the primitives are the predicates of the two types of variables general recursive in number-theoretic functions $\psi_{1}, \cdots, \psi_{l}\left({ }^{4}\right)$, a predicate $P$ so definable (so definable without use of function quantifiers) we call analytic (arithmetical) in $\psi_{1}, \cdots, \psi_{l}(l \geqq 0)$. (Cf. IM p. 285.)

The notions extend to the case $P$ is replaced by a function $\phi$, via the representing predicate of $\phi$ (IM p. 199); and to the case any $\psi$ is replaced by a predicate $Q$, via the representing function of $Q$.

2.2 In the definitions just given, we have taken the primitives to be exactly the predicates general recursive in $\psi_{1}, \cdots, \psi_{l}$. Now we shall observe that equivalently in these definitions we could choose the primitives in some other ways.

Thus the class of the allowed primitives can be enlarged to include also the functions general recursive in $\psi_{1}, \cdots, \psi_{l}$. This is trivial, since predicates recursive in $\psi_{1}, \cdots, \psi_{l}$ result by the substitution of functions recursive in $\psi_{1}, \cdots, \psi_{l}$ into predicates recursive in $\psi_{1}, \cdots, \psi_{l}$, which is the only way the additional primitives could be used.

2.3 Next we observe that we can restrict the predicates general recursive in $\psi_{1}, \cdots, \psi_{l}$ to be primitive recursive predicates of number variables only, provided that the same time we allow their function variables and $\psi_{1}, \cdots, \psi_{l}$ to enter as primitives $\left({ }^{6}\right)$.

For adding the function variables and $\psi_{1}, \cdots, \psi_{l}$ gives no more predicates, by 2.2 .

Then omitting all but these and primitive recursive predicates of number variables only removes. none, since any predicate $R$ general recursive in $\psi_{1}, \cdots, \psi_{l}$ can be expressed in terms of the remaining primitives. For example with $l=1$ and no function variables, if $R^{\psi}(a)$ is general recursive in $\psi(b)$, then using IM Theorem VI* (a) with its proof from Theorem IV* (6) pp. 281, 284, 292,

$$
\begin{aligned}
& R^{\psi}(a) \equiv(E x) T_{1}^{\psi}(f, a, x) \equiv(E x) T_{1}^{1}(\tilde{\psi}(x), f, a, x) \\
& \equiv(E x)(E v)\left\{\tilde{\psi}(x)=v \& T_{1}^{1}(v, f, a, x)\right\} \\
& \equiv(E x)(E v)\left\{v=\prod_{i<x} p_{i}^{(v)_{i}} \&(i)_{i<x}\left[\psi(i)=(v)_{i}\right] \& T_{1}^{1}(v, f, a, x)\right\} .
\end{aligned}
$$

2.4 Continuing from 2.3 , a function $\psi(b)$ can be replaced as primitive by its representing predicate $\psi(b)=w$. For adding $\psi(b)=w$ obviously gives no more predicates. Then omitting $\psi(b)$ removes none, since e.g. in the example of 2.3 we can replace $\psi(i)=(v)_{i}$ by $(E w)\left[\psi(i)=w \& w=(v)_{i}\right]$.

2.5 Again continuing from 2.3, a fun ${ }^{-}$ion $\psi(b)$ which is the representing function of a predicate $Q(b)$ can be replaced as primitive by $Q(b)$. For adding $Q(b)$ as primitive gives no more predicates, since $Q(b) \equiv \psi(b)=0$. Then omit-

${ }^{(6)}$ One further step leads to the more usual definition of "arithmetical" for numbertheoretic predicates, in which the primitives are $+, \cdot,=, 0$ and 1 (cf. IM $\$ \$ 48,49$ ). For "analytic", the primitives are then still further reducible to $=, 0$ and +1 by using Lorenzen's method [12] to define the representing predicates of + and $\cdot$ 
ting $\psi(b)$ removes none, since e.g. in the example of 2.3 we can replace $\psi(i)=(v)_{i}$ by

$$
\left\{Q(i) \&(v)_{i}=0\right\} \vee\left\{\bar{Q}(i) \&(v)_{i}=1\right\} .
$$

2.6 A predicate $P$ arithmetical in arithmetical predicates or functions $\Psi$ (in predicates or functions $\Psi$ arithmetical in $\Theta$ ) is arithmetical (arithmetical in $\Theta)$. Similarly with analytic.

For we can use 2.3-2.5 so that the primitives for $P$ become, besides primitive recursive predicates and function variables, the predicates among $\Psi$ themselves and the representing predicates of the functions among $\Psi$, after which the expressions for those predicates from the primitives for them can be introduced.

3. Alterations of quantifiers. 3.1 Starting from an expression under 2.1 for an analytic predicate, the quantifiers can be advanced to obtain a prenex form. We can then contract adjacent number quantifiers of like kind (IM p. 285 (17) and (18)), leaving the scope of the prefix recursive. So every arithmetical predicate, e.g. of one number variable $a$, is expressible in one of the forms

$$
R(a) \begin{array}{rrrr}
(E x) R(a, x) & (x)(E y) R(a, x, y) & (E x)(y)(E z) R(a, x, y, z) & \ldots \\
(x) R(a, x) & (E x)(y) R(a, x, y) & (x)(E y)(z) R(a, x, y, z) & \ldots
\end{array}
$$

where the $R$ for each is general recursive (IM Theorem VII (d) p. 285, or with function variables VII* (d) p. 292).

In the remainder of this section we obtain a corresponding list of forms for the analytic predicates.

3.2 Corresponding to IM p. 285 (17) and (18), we now have

$$
\begin{gathered}
\left(E \alpha_{0}\right) \cdots\left(E \alpha_{m}\right) A\left(\alpha_{0}, \cdots, \alpha_{m}\right) \equiv(E \alpha) A\left(\lambda t(\alpha(t))_{0}, \cdots, \lambda t(\alpha(t))_{m}\right), \\
\left(\alpha_{0}\right) \cdots\left(\alpha_{m}\right) A\left(\alpha_{0}, \cdots, \alpha_{m}\right) \equiv(\alpha) A\left(\lambda t(\alpha(t))_{0}, \cdots, \lambda t(\alpha(t))_{m}\right) .
\end{gathered}
$$

Thus adjacent function quantifiers of like kind can also be contracted.

3.3 We can indeed contract adjacent quantifiers of the same kind mixed as to their types, by first raising the type of the number quantifiers thus $\left({ }^{7}\right)$,

$$
\begin{gathered}
(E x) A(x) \equiv(E \alpha) A(\alpha(0)), \\
(x) A(x) \equiv(\alpha) A(\alpha(0)) .
\end{gathered}
$$

3.4 A function quantifier can be advanced across a number quantifier of the opposite kind. For

$$
\begin{aligned}
& (x)(E \alpha) A(x, \alpha) \equiv(E \alpha)(x) A\left(x, \lambda t \alpha\left(2^{x} \cdot 3^{t}\right)\right), \\
& (E x)(\alpha) A(x, \alpha) \equiv(\alpha)(E x) A\left(x, \lambda t \alpha\left(2^{x} \cdot 3^{t}\right)\right) .
\end{aligned}
$$

Here (6) follows from (5) by the method of proof of duality in logic; namely,

( $\left.{ }^{7}\right)$ Or directly; e.g. $(x)(y)(z)(\alpha) A(x, y, z, \alpha) \equiv(\alpha) A(\alpha(0), \alpha(1), \alpha(2), \lambda t \alpha(t+3))$. 
we apply (5) to $\bar{A}$ instead of to $A$, negate both sides of the resulting equivalence, and move the negations inward $\left({ }^{8}\right)$.

3.5 A function quantifier applied directly to a recursive predicate can be reduced in type to a number quantifier. For (cf. 2.3, changing $R^{\psi}(a)$ to $R(\alpha, a)$, and choosing an $f$ independent of $\alpha$, as we can do by the uniformity requirement in 1.2 with the method of proof of IM Theorem IV*),

(7) $(E \alpha) R(\alpha, a) \equiv(E \alpha)(E x) T_{1}^{1}(\tilde{\alpha}(x), f, a, x) \equiv(E x) T_{1}^{1}\left(\prod_{i<(x)_{0}} p_{i}^{(x)_{i+1}}, f, a,(x)_{0}\right)$,

$$
(\alpha) R(\alpha, a) \equiv(\alpha)(x) \bar{T}_{1}^{1}(\tilde{\alpha}(x), g, a, x) \equiv(x) \bar{T}_{1}^{1}\left(\prod_{i<(x)_{0}} p_{i}^{(x)_{i+1}}, g, a,(x)_{0}\right) .
$$

3.6 Theorems 1 and 2 apply to predicates of any fixed list of $m+n$ variables $\eta_{1}, \cdots, \eta_{m}, a_{1}, \cdots, a_{n}(m, n \geqq 0)$, but for notational convenience we state them for one variable $a(m=0, n=1)$.

THEOREM 1 (PART 1). Each analytic predicate is expressible in one of the following forms where $A$ is arithmetical and each $R$ is recursive:

$$
A(a) \begin{array}{lll}
(\alpha)(E x) R(a, \alpha, x) & (E \alpha)(\beta)(E x) R(a, \alpha, \beta, x) & \cdots \\
(E \alpha)(x) R(a, \alpha, x) & (\alpha)(E \beta)(x) R(a, \alpha, \beta, x) & \cdots
\end{array}
$$

Proof. After bringing an expression for an analytic predicate under 2.1 to prenex form (3.1), we apply the following steps, throughout which the scope of the prefix remains recursive. The example following illustrates the steps.

SteP 1. Contract adjacent quantifiers of like kind (3.1-3.3). Now the quantifiers alternate in kind (and will at the conclusion of each subsequent step).

STEP 2. If the rightmost quantifier is a function quantifier $\left({ }^{9}\right)$, change it to a number quantifier (3.5). (If no function quantifier remains, then we are done, as we have the first form $A(a)$ of $\left(\mathrm{b}_{1}\right)$, and indeed this $A(a)$ is already in one of the forms (a) 3.1.)

Step 3. Now consider the number quantifiers standing to the right of the rightmost function quantifier. If they are more than one, remove all but the leftmost one, thus. Change the rightmost number quantifier of the same kind as the rightmost function quantifier to a function quantifier (3.3), and advance it (3.4), after each advance contracting adjacent quantifiers of like kind $(3.1-3.3)\left({ }^{10}\right)$.

STEP 4. Finally consider each group of number quantifiers included be-

(8) Similarly to (5), $(x)(y)(E \alpha) A(x, y, \alpha) \equiv(E \alpha)(x)(y) A\left(x, y, \lambda t \alpha\left(2^{x} \cdot 3^{y} \cdot 5^{t}\right)\right)$; etc.

( 9 ) As will appear from Step 3 , if the second rightmost function quantifier is of the same kind, we may omit Step 2.

( $\left.{ }^{10}\right)$ Another method: e.g. $(E \alpha)(x)(E y)(z)(E t) A(\alpha, x, y, z, t) \equiv(E \alpha)(E \beta)(E \gamma)(x)(z) A(\alpha, x$, $\beta(x), \quad z, \quad \gamma(x, z)) \equiv(E \alpha)(E \beta)(E \gamma)(x)(z) A\left(\alpha, x, \quad \beta(x), \quad z, \quad \gamma\left(2^{x} \cdot 3^{z}\right)\right) \equiv(E \alpha)(x) A\left(\lambda t(\alpha(t))_{0}, \quad(x)_{0}\right.$, $\left.\left(\alpha\left((x)_{0}\right)\right)_{1},(x)_{1},\left(\alpha\left(2^{(x)_{0}} \cdot 3^{(x)_{1}}\right)\right)_{2}\right)$. 
tween function quantifiers or to the left of the leftmost function quantifier. Remove successively each such group of number quantifiers, and if function quantifiers of like kind are brought together thereby contract them. To remove such a group of number quantifiers, advance the function quantifier on the right and perform contractions, as in the last part of Step 3.

EXAMPLE. Suppose given the prefix numbered 1 applied directly to a recursive predicate (say of $a$ and the variables in that prefix). The whole expression is then equivalent to ones with the successive new prefixes; each time the scope is altered but remains a recursive predicate of $a$ and the variables in the prefix.

1. $(E u)(v)(E w)(E \alpha)(x)(y)(E z)(\beta)$.

2. $(E u)(v) \quad(E \alpha)(x) \quad(E z)(\beta)-(3)$ and (1), IM (18) (end Step 1).

3. $(E u)(v) \quad(E \alpha)(x) \quad(E z)(y)-(8)$ (end Step 2).

4. $(E u)(v) \quad(E \alpha)(x) \quad(E \beta)(y)-(3)$.

5. $(E u)(v) \quad(E \alpha)(E \beta) \quad(x)(y)-(5)$.

6. $(E u)(v) \quad(E \alpha) \quad(x) \quad-(1)$, IM (18) (end Step 3).

7. $(E u)(E \alpha)$

$(v)$

$(x)-(5)$.

8. $\quad(E \alpha)$

$(x) \quad-(3)$ and (1), IM (18) (end Step 4).

Remark. Merely to prove the theorem we could proceed more directly, say by raising all number quantifiers to function quantifiers, and introducing the additional quantifiers required redundantly (as we could have done for (a) also); but Steps 1-4 lead to the simplest prefix obtainable in general from a given prefix (cf. Corollary Theorem 3 ).

3.7 TheOREM 1 (PART 2). Equivalently the forms can be written as follows where the $A$ for each is arithmetical (i.e. the same predicates are expressible in a given form of $\left(\mathrm{b}_{1}\right)$ as in the respective form of $\left.\left(\mathrm{b}_{2}\right)\right)$ :

$$
A(a) \begin{array}{rll}
(\alpha) A(a, \alpha) & (E \alpha)(\beta) A(a, \alpha, \beta) & \cdots \\
(E \alpha) A(a, \alpha) & (\alpha)(E \beta) A(a, \alpha, \beta) & \cdots
\end{array}
$$

Proof. Thus e.g. a predicate of the form $(E \alpha)(x) R(a, \alpha, x)$ is of the form $(E \alpha) A(a, \alpha)$, since for $R$ recursive $(x) R(a, \alpha, x)$ is arithmetical; while given any predicate of the form $(E \alpha) A(a, \alpha)$, by first putting $A(a, \alpha)$ in one of the forms (a) 3.1, and then applying Step 1, and Step 3 (or introducing a redundant $(x)$ ), we get an expression for it of the form $(E \alpha)(x) R(a, \alpha, x)$.

3.8 THEOREM 1 (PART 3). If the bound function variables $\alpha, \beta, \cdots$ are changed to ones $\alpha^{*}, \beta^{*}, \cdots$ ranging only over functions taking only 0 and 1 as values (i.e. in effect, to set variables), the forms $\left(\mathrm{b}_{1}\right)$ become equivalently (with $R$ recursive)

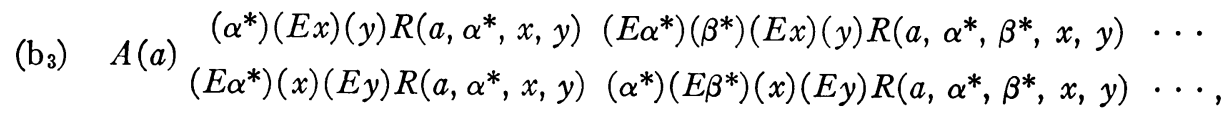


and $\left(\mathrm{b}_{2}\right)$ remain otherwise unchanged (call them then $\left(\mathrm{b}_{4}\right)$ ).

Proof. For example (starting from one of $\left.\left(\mathrm{b}_{4}\right)\right),\left(E \alpha^{*}\right)\left(\beta^{*}\right) A\left(a, \alpha^{*}, \beta^{*}\right)$ $\equiv(E \alpha)\{(t)[\alpha(t) \leqq 1] \&(\beta)\{(t)[\beta(t) \leqq 1] \rightarrow A(a, \alpha, \beta)\}\}$, which latter expression reduces to the corresponding form $(E \alpha)(\beta)(E x) R(a, \alpha, \beta, x)$ of $\left(\mathrm{b}_{1}\right)$ by (the method of proof of) Part 1.

To write e.g. $(E \alpha)(\beta)(E x) R(a, \alpha, \beta, x)$ in the corresponding form $\left(E \alpha^{*}\right)\left(\beta^{*}\right)(E x)(y) R\left(a, \alpha^{*}, \beta^{*}, x, y\right)$ of $\left(\mathrm{b}_{3}\right)$, we proceed thus. Using IM Theorem IV* pp. 281, 291, 292, for some $f$ (independent of $\alpha$, using 1.2), $(E x) R(a, \alpha, \beta, x) \equiv(E x) T_{1}^{1,1}(\tilde{\alpha}(x), \tilde{\beta}(x), f, a, x)$. Let $C(\gamma, x, y) \equiv \gamma(x) \leqq 1$ $\left.\&\left[\gamma(x)=0 \rightarrow x=2^{(x)_{0}} \cdot 3^{(x)}\right)_{1} \&(t)_{t<(x)_{1}} \gamma\left(2^{(x)_{0}} \cdot 3^{t}\right)=1\right] \quad \& \quad(s)_{s<x}\left[\gamma\left(2^{s} \cdot(3 \exp \right.\right.$ $\left.\left.\left.\prod_{i<s} p_{i}^{(\nu)_{i}}\right)\right)=0\right]$. Then the predicate $C$ is recursive, and $(x)(E y) C(\gamma, x, y)$ $\equiv\left\{\gamma\right.$ is the representing function of $x=2^{(x)_{0}} \cdot 3^{\widetilde{\alpha}\left((x)_{0}\right)}$, for some function $\left.\alpha\right\}$, in which case $\alpha(x)=\left(\mu y\left[\gamma\left(2^{x+1} \cdot 3^{y}\right)=0\right]\right)_{x}$. Now $(E \alpha)(\beta)(E x) R(a, \alpha, \beta, x)$ $\equiv\left(E \alpha^{*}\right)\left\{(x)(E y) C\left(\alpha^{*}, x, y\right) \&(\beta)(E x)(E y)\left[\alpha^{*}\left(2^{x} \cdot 3^{y}\right)=0 \quad \& \quad T_{1}^{1,1}(y, \tilde{\beta}(x)\right.\right.$, $f, a, x)]\}$, which latter reduces to the form $\left(E \alpha^{*}\right)(\beta)(E x) S\left(a, \alpha^{*}, \beta, x\right)$ with $S$ recursive by Part 1 . By duality it will suffice now to reduce $(E \beta)(x) \bar{S}\left(a, \alpha^{*}\right.$, $\beta, x)$ to the form $\left(E \beta^{*}\right)(x)(E y) \bar{R}\left(a, \alpha^{*}, \beta^{*}, x, y\right)$. But using Theorem IV* again, $(E \beta)(x) \bar{S}\left(a, \quad \alpha^{*}, \quad \beta, \quad x\right) \equiv(E \beta)(x) \bar{T}_{1}^{1,1}\left(\tilde{\alpha}^{*}(x), \quad \tilde{\beta}(x), \quad g, \quad a, \quad x\right) \equiv$ $\left(E \beta^{*}\right)\left\{(x)(E y) C\left(\beta^{*}, x, y\right) \&(x)(E y)\left[\beta^{*}\left(2^{x} \cdot 3^{y}\right)=0 \& \bar{T}_{1}^{1,1}\left(\tilde{\alpha}^{*}(x), y, g, a, x\right)\right]\right\}$, which latter reduces to the form $\left(E \beta^{*}\right)(x)(E y) \bar{R}\left(a, \alpha^{*}, \beta^{*}, x, y\right)$ by 3.1 .

4. The enumeration and hierarchy theorems. 4.1 Next we give the analogs for $\left(b_{1}\right)$ of the enumeration and hierarchy theorems for (a) (IM Theorems IV, V, IV*, V* pp. 281, 283, 292; or [5])( $\left.{ }^{2}\right)$.

TheOREM 2. To each of the forms of Theorem 1 after the first, there is an enumerating predicate of that form for the predicates of that form. For example: Given any recursive predicate $R(a, \alpha, x)$, there are numbers $f$ and $g$ such that

$$
\begin{aligned}
& (\alpha)(E x) R(a, \alpha, x) \equiv(\alpha)(E x) T_{1}^{\alpha}(f, a, x), \\
& (E \alpha)(x) R(a, \alpha, x) \equiv(E \alpha)(x) \bar{T}_{1}^{\alpha}(g, a, x) .
\end{aligned}
$$

Thus $(\alpha)(E x) T_{1}^{\alpha}(z, a, x)$ and $(E \alpha)(x) \bar{T}_{1}^{\alpha}(z, a, x)$ are enumerating predicates for the forms $(\alpha)(E x) R(a, \alpha, x)$ and $(E \alpha)(x) R(a, \alpha, x)$, respectively.

Proof. Essentially as before; thus (9) comes from IM Theorem IV* (6) pp. 281, 292 for $l=n=1$ and $f$ independent of $\alpha$ by quantifying $\alpha$.

COROllary. The class of the predicates expressible in a given one of the forms $\left(b_{1}\right)$ (or $\left.\left(b_{3}\right)\left({ }^{11}\right)\right)$ after the first is the same whether a general recursive $R$ or only a primitive recursive $R$ be allowed.

4.2 The hierarchy theorem and its corollary apply to any fixed list of $m+n$ variables $\eta_{1}, \cdots, \eta_{m}, a_{1}, \cdots, a_{n}$ with $m+n>0$.

(11) By the proof of Theorem 1 Part 3 from IM Theorem IV*. 
THEOREM 3. To each of the forms of Theorem 1 after the first, there is a predicate expressible in that form but not in the dual form (a fortiori, not in any of the forms with fewer quantifiers).

Proof for $n>0$. From the enumeration theorem as before (IM pp. 282$284)$; e.g. $(\alpha)(E x) T_{1}^{\alpha}(a, a, x)$ is not expressible in the form $(E \alpha)(x) R(a, \alpha, x)$ with $R$ recursive.

Proof for $n=0$. Then $m>0$, as $m+n>0$. For example, $(\alpha)(E x) T_{0}^{\eta, \alpha}(\eta(0), x)$ is not expressible in the form $(E \alpha)(x) R(\eta, \alpha, x)$ with $R$ recursive. For by the enumeration theorem, given such an $R$, there is a number $g$ such that $(E \alpha)(x) R(\eta, \alpha, x) \equiv(E \alpha)(x) \bar{T}_{0}^{\eta, \alpha}(g, x)$. Substituting for $\eta$ any function $\eta_{1}$ with $\quad \eta_{1}(0)=g, \quad(E \alpha)(x) R\left(\eta_{1}, \quad \alpha, \quad x\right) \equiv(E \alpha)(x) \bar{T}_{0}^{\eta_{1}, \alpha}\left(\eta_{1}(0), \quad x\right) \equiv \overline{(\alpha)}(E x)$ $T_{0}^{\eta_{1}, \alpha}\left(\eta_{1}(0), x\right) \not \equiv(\alpha)(E x) T_{0}^{\eta_{1}, \alpha}\left(\eta_{1}(0), x\right)$.

COROLlaRy. To any prenex form with recursive scope, there is a predicate of that form which is expressible in no other of the forms $\left(\mathrm{b}_{1}\right)$ (and for the first form of $\left(\mathrm{b}_{1}\right)$, in no other of the forms (a)) than the one to which Steps 1-4 lead except ones with more quantifiers.

Thus, since any prenex form with recursive scope not in (a) or $\left(b_{1}\right)$ is simplified by Steps 1-4, Steps 1-4 lead to the unique simplest prefix possible in general.

Proof. The net effect of each of the four steps is to suppress quantifiers or to depress a function quantifier to a number quantifier. These steps are reversible, so the predicate of Theorem 3 (of Theorem $\mathrm{V}$ or $\mathrm{V}^{*}$ ) for a given form of $\left(b_{1}\right)$ (of $(a)$ ) after the first can be worked back into any prenex form which Steps 1-4 would reduce to that form.

4.3 We now parallel a theorem, the case of which for arithmetical predicates was emphasized by Post. He talked of sets rather than predicates, saying there is a complete recursively enumerable set $K$ [19], or more generally to any set $S$ a complete $S$-canonical set $S^{\prime}$ [20].

We start with the case of predicates of a fixed list of $m+n$ variables $\eta_{1}, \cdots, \eta_{m}, a_{1}, \cdots, a_{n}$ with $m+n>0$.

ThEOREM 4 (PART 1). To each of the forms of Theorem 1 after the first, there is a particular predicate of that form such that any predicate of the form is expressible by substitution of a recursive function for one of its variables $\left({ }^{12}\right)$.

Proof for $n>0$. Essentially as before (IM p. 343 Example 2); e.g. for the form $(\alpha)(E x) R(a, \alpha, x)$, using the same predicate $(\alpha)(E x) T_{1}^{\alpha}(a, a, x)$ as for Theorem $3\left({ }^{13}\right),(\alpha)(E x) R(a, \alpha, x) \equiv(\alpha)(E x) T_{1}^{\alpha}\left(S_{1}^{1,1}(e, a), S_{1}^{1,1}(e, a), x\right)$ when $e$

${ }^{(12)}$ And indeed is 1-1 reducible to it (Post [19]), i.e. the function substituted takes on distinct values for distinct arguments.

(13) Any predicate for Theorem 4 is necessarily one for Theorem 3, by Theorem 3. At least in the arithmetical case, a predicate for Theorem 3 need not be one for Theorem 4 , an example for the form $(E x) R(a, x)$ being $a \in S$ where $S$ is simple (Post [19, pp. 298-304]). 
is any uniform Gödel number from $\alpha$ (i.e. one independent of $\alpha$ ) of $\lambda a z \mu x$ $R(a, \alpha, x)$.

Proof for $n=0$. Consider e.g. the form $(\alpha)(E x) R(\eta, \alpha, x)$, with the same predicate $(\alpha)(E x) T_{0}^{\eta, \alpha}(\eta(0), x)$ as for Theorem $3\left({ }^{14}\right)$. By Theorem 2 with 1.3, $(\alpha)(E x) R(\lambda t \eta(t+1), \alpha, x) \equiv(\alpha)(E x) T_{0}^{\eta, \alpha}(f, x)$. So if we let

$$
\left\{\begin{aligned}
\phi(0, \eta) & =f \\
\phi(t+1, \eta) & =\eta(t)
\end{aligned}\right.
$$

then $(\alpha)(E x) T_{0}^{\lambda t \phi(t, \eta), \alpha}(\{\lambda t \phi(t, \eta)\}(0), x) \equiv(\alpha)(E x) R(\eta, \alpha, x)$.

THEOREM 4 (PART 2). To each of the forms of Theorem 1 after the first, there is a particular predicate of that form with one number variable (one function variable) such that any predicate of the form with any number of number variables only (of number and function variables) is expressible by substitution of a recursive function for its variable $\left({ }^{15}\right)$.

Proof. By applying to the free variables the principles used in contracting adjacent quantifiers of like kind (3.1-3.3), each predicate of more than one variable is expressible by substitution in a one-variable predicate, after which Part 1 is applicable. For example, $P\left(a_{0}, a_{1}\right) \equiv Q\left(2^{a_{0}} \cdot 3^{a_{1}}\right)$, if $Q(a) \equiv P\left((a)_{0},(a)_{1}\right)$; $P(a) \equiv Q(\lambda t a), \quad$ if $Q(\eta) \equiv P(\eta(0)) ; \quad P\left(\eta_{0}, \quad \eta_{1}\right) \equiv Q\left(\lambda t 2^{\eta_{0}(t)} \cdot 3^{\eta_{1}(t)}\right), \quad$ if $Q(\eta) \equiv$ $P\left(\lambda t(\eta(t))_{0}, \lambda t(\eta(t))_{1}\right)$.

When the context indicates reference to the analytic hiearchy $\left(b_{1}\right)$ or $\left(\mathrm{b}_{2}\right)$, by $k$-quantifier forms we mean those with $k$ function quantifiers, and by $k$-quantifier predicates predicates expressible in one of the $k$-quantifier forms. (When the reference is to (a), $k$ quantifiers means $k$ number quantifiers.)

Corollary. For each $k(k=0,1,2, \cdots)$, there is a particular $k+1$ quantifier predicate of one number variable in which each $k+1$-quantifier predicate of any number of number variables only is recursive.

Proof. Take the predicate of the theorem Part 2 for one of the $k+1$ quantifier forms. The predicates of the other $k+1$-quantifier form are also recursive in it, since they are negations of predicates of that form (IM \#D p. 228).

5. The notion "arithmetical" in relation to the hierarchy. 5.1 In the arithmetical hierarchy (a), the predicates expressible in both 1-(number-) quantifier forms are exactly the general recursive predicates (Kleene [5], Post [19], Mostowski [15], IM Theorem VI p. 284); and more generally, the

(14) Another predicate (suggested by IM p. 346 Example 5) is $(\alpha)(E x)[\eta(\bar{\alpha}(x))=0](2)$. For let $\tau(v, w, z)$ be the representing function of $T_{0}^{1.1}(v, w, z)$. Given $R$, choose $f$ by Theorem 2 so that $(\alpha)(E x) R(\eta, \alpha, x) \equiv(\alpha)(E x) T_{0}^{\eta, \alpha}(f, x) \equiv(\alpha)(E x) T_{0}^{1,1}(\eta(x), \bar{\alpha}(x), f)$. Let $\phi(t, \quad \eta)$ $=\tau(\bar{\eta}(\operatorname{lh}(t)), t, f)$. Then $(\alpha)(E x)[\{\lambda t \phi(t, \eta)\}(\bar{\alpha}(x))=0] \equiv(\alpha)(E x) R(\eta, \alpha, x)$.

(15) Or there may be a fixed list of additional variables as parameters. 
predicates expressible in both $k+1$-quantifier forms are exactly those general recursive in $k$-quantifier predicates (Post [20], IM Theorem XI p. 293).

Despite the formal similarity of $\left(b_{2}\right)$ to $(a)$, this theorem does not carry over completely. As we shall see $(5.2,5.3)$, a predicate arithmetical in $k$ (function-)quantifier predicates (of number variables only $\left({ }^{4}\right)$ ) is expressible in both $k+1$-quantifier forms, but not always conversely.

The situation is thus analogous to what one has by reading "primitive recursive" in place of "(general) recursive" in the arithmetical hierarchy (a), in which form the arithmetical hierarchy theorem could have been stated (though it was not) before the advent of the notion "general recursive" (by IM Corollary Theorem IV p. 282). The problem would then have existed to obtain a characterization, independent of the hierarchy, of the predicates expressible in both 1-quantifier forms, or more generally of those expressible in both $k+1$-quantifier forms. We now have such a problem for the hierarchy $\left(\mathrm{b}_{2}\right)$.

For simplicity, we begin in this section by treating the relation between the predicates expressible in both $k+1$-quantifier forms and the predicates arithmetical in $k$-quantifier predicates. Then, after preliminaries in $\S 6$, we shall show in $\S 8$ how similar results hold for a certain very great extension of "arithmetical", which permits a conjecture on the above problem (8.6).

5.2 Theorem 5. Every predicate arithmetical in predicates expressible in both $k+1$-quantifier forms is expressible in both $k+1$-quantifier forms.

Proof. For example (taking $k=n=1$ and $m=0$ ), consider a predicate $P(a)$ arithmetical in predicates $Q_{1}, \cdots, Q_{s}$ each expressible in both 2-quantifier forms.

Let an expression under 2.1 with 2.3 and 2.5 for $P(a)$ in terms of $Q_{1}, \cdots, Q_{s}$ be brought to a prenex form, consisting of number quantifiers applied to a scope composed by operations of the propositional calculus applied to primitive recursive predicates and occurrences of $Q_{1}, \cdots, Q_{s}$ with various variables in their argument positions. Without loss of generality we can assume there is at least one such occurrence.

Let an occurrence in this scope of a $Q$ be called positive or negative, according as it lies in the $A$ of an even or odd number of parts of the form $A \rightarrow B$ or $\bar{A}$.

Let each positive occurrence of a $Q$ be replaced by the 2-quantifier expression for it with the prefix $(E \alpha)(\beta)(E x)$, and each negative occurrence by the one with the prefix $(\alpha)(E \beta)(x)$.

Now advance the quantifiers $(E \alpha)$ and $(\alpha)$ to the front of the scope (of the number-theoretic prefix of the prenex form), where they all become existential quantifiers. Next advance the quantifiers $(\beta)$ and $(E \beta)$, which all become generality quantifiers. Finally advance the quantifiers $(E x)$ and $(x)$, which become existential quantifiers. 
Now application of 3.6 Steps 1 and 4 to the resulting prefix will lead to a prefix of the form $(E \alpha)(\beta)(E x)$.

We obtain the other 2-quantifier form similarly using $(\alpha)(E \beta)(x)$ for positive, and $(E \alpha)(\beta)(E x)$ for negative, occurrences of $Q_{1}, \cdots, Q_{8}$.

COROLLARY. Every predicate arithmetical in k-quantifier predicates (or these and also predicates expressible in both $k+1$-quantifier forms) is expressible in both $k+1$-quantifier forms.

Proof. A $k$-quantifier predicate is expressible in either $k+1$-quantifier form by introducing appropriately a redundant quantifier (for $k=0$, this gives a form of $\left(b_{2}\right)$ ).

5.3 THEOREM 6. There is a predicate $P(a)$ expressible in both $k+1$-quantifier forms which is not arithmetical in k-quantifier predicates and in which every predicate $A(a)$ arithmetical in $k$-quantifier predicates is recursive.

Proof. Let $Q(a)$ be $a=a$ for $k=0$, and for $k>0$ be a $k$-quantifier predicate given by Corollary Theorem 4 in which every $k$-quantifier predicate (of number variables only) is recursive.

Consider the predicates $Q(a),(E x) T_{1}^{Q}(a, a, x),(x)(E y) T_{2}^{Q}(a, a, x, y), \cdots$; call them $N_{0}^{Q}(a), N_{1}^{Q}(a), N_{2}^{Q}(a), \cdots$. But $T_{1}^{Q}, T_{2}^{Q}, \cdots$ are primitive recursive in $Q$; so $N_{i}^{Q}(i=0,1,2, \cdots)$ is arithmetical in $Q$, and hence by Corollary Theorem 5 (for $k=0, l=1$; cf. end 1.2) expressible in both 1-quantifier forms relative to $Q$. Hence by Theorem $2, N_{i}^{Q}(a) \equiv(\alpha)(E x) T_{1}^{Q, \alpha}\left(f_{i}, a, x\right)$ $\equiv(E \alpha)(x) \bar{T}_{1}^{Q, \alpha}\left(g_{i}, a, x\right)$.

The numbers $f_{i}$ and $g_{i}$ are determined effectively by the proofs of the cited theorems, so by Church's thesis (cf. IM $\S 62$ ) it is to be expected that $f_{i}=\eta(i)$ and $g_{i}=\theta(i)$ with general recursive $\eta$ and $\theta\left({ }^{16}\right)$.

We let $P(a) \equiv N_{(a)_{1}}^{Q}\left((a)_{0}\right), \quad$ so $P(a) \equiv(\alpha)(E x) T_{1}^{Q, \alpha}\left(\eta\left((a)_{1}\right), \quad(a)_{0}, \quad x\right) \equiv$ $(E \alpha)(x) \bar{T}_{1}^{Q, \alpha}\left(\theta\left((a)_{1}\right),(a)_{0}, x\right)$.

Now consider any predicate $A(a)$ arithmetical in $k$-quantifier predicates, and hence in $Q$. Then $A(a)$ is expressible in one of the forms (a) relative to $Q$; suppose e.g. $A(a) \equiv(x)(E y) R^{Q}(a, x, y)$ with $R^{Q}$ recursive in $Q$. Then using IM p. 343 Example 2 for $l=1$, for some recursive $\phi, A(a) \equiv(x)(E y) R^{Q}(a, x, y)$ $\equiv(x)(E y) T_{2}^{Q}(\phi(a), \phi(a), x, y) \equiv N_{2}^{Q}(\phi(a)) \equiv P\left(2^{\phi(a)} \cdot 3^{2}\right)$. Thus $A(a)$ is recursive in $P(a)$.

Next suppose $P(a)$ were arithmetical in $k$-quantifier predicates. Then $(E x) T_{1}^{P}(a, a, x)$ would be, and so by the preceding would be recursive in $P$, which is absurd (IM Corollary Theorem XI* p. 295).

Finally, for $k=0 P(a)$ is already expressed in both 1-quantifier forms, while for $k>0$ we can argue thus. As predicate of $\alpha$ and $a,(E x) T_{1}^{Q, \alpha}\left(\eta\left((a)_{1}\right)\right.$, $\left.(a)_{0}, x\right)$ is arithmetical in $Q$, so by Corollary Theorem 5 it is expressible in both $k+1$-quantifier forms, in particular in the one with generality first. Using this in $P(a) \equiv(\alpha)(E x) T_{1}^{Q, \alpha}\left(\eta\left((a)_{1}\right),(a)_{0}, x\right)$, and contracting by (2), we

(16) In fact there are primitive recursive $\eta$ and $\theta$, as will be shown in 9.8 . 
obtain a $k+1$-quantifier expression for $P(a)$ with generality first. The other $k+1$-quantifier expression for $P(a)$ is obtained similarly.

5.4 TheORem 7. To any predicate $Q(a)$ expressible in both 1-quantifier forms, there is a recursive $R$ such that $(E \alpha)(x) R(\alpha, x)$ is true but $(x) R(\alpha, x)$ is false for every $\alpha$ recursive in $Q(k=0)$.

To any predicate $Q(a)$ expressible in both $k+1$-quantifier forms, there is a $k$-quantifier predicate $B(\alpha)$ with generality first such that $(E \alpha) B(\alpha)$ is true but $B(\alpha)$ is false for every $\alpha$ recursive in $Q(k=1,2,3, \cdots)$.

Proof. We begin by discussing predicates $P(a)$ expressible in both 1-quantifier forms relative to $Q$. So suppose $P(a) \equiv(E \alpha)(x) A^{Q}(a, \alpha, x) \equiv(\alpha)(E x)$ $B^{Q}(a, \alpha, x)$ where $A^{Q}$ and $B^{Q}$ are recursive in $Q$. Then $\bar{P}(a) \equiv(E \alpha)(x)$ $\bar{B}^{Q}(a, \alpha, x)$. Now $(a)\{P(a) \vee \bar{P}(a)\}$ is true. This we can transform thus:

$$
\begin{aligned}
& \text { (a) }\{P(a) \vee \bar{P}(a)\} \equiv(a)\left\{(E \alpha)(x) A^{Q}(a, \alpha, x) \vee(E \alpha)(x) \bar{B}^{Q}(a, \alpha, x)\right\} \\
& \equiv(a)(E \alpha)(x)(y)\left\{A^{Q}(a, \alpha, x) \vee \bar{B}^{Q}(a, \alpha, y)\right\} \\
& \equiv(E \alpha)(a)(x)(y)\left\{A^{Q}\left(a, \lambda t \alpha\left(2^{a} \cdot 3^{t}\right), x\right) \vee \bar{B}^{Q}\left(a, \lambda t \alpha\left(2^{a} \cdot 3^{t}\right), y\right)\right\} \\
& \equiv(E \alpha)(x)\left\{A ^ { Q } ( ( x ) _ { 0 } , \lambda t \alpha ( 2 ^ { ( x ) _ { 0 } \cdot 3 ^ { t } } ) , ( x ) _ { 1 } ) \vee \overline { B } ^ { Q } \left((x)_{0}, \lambda t \alpha\left(2^{\left.\left.\left.(x)_{0} \cdot 3^{t}\right),(x)_{2}\right)\right\}}\right.\right.\right.
\end{aligned}
$$

Call the last expression $(E \alpha)(x) R^{Q}(\alpha, x)$.

Now let $\alpha_{1}$ be any particular function $\alpha$ which makes $(x) R^{Q}(\alpha, x)$ true. For any number $a$, let $\alpha_{a}$ abbreviate $\lambda t \alpha_{1}\left(2^{a} \cdot 3^{t}\right)$. By reversing part of the above transformation, we obtain then $(a)\left\{(x) A^{Q}\left(a, \alpha_{a}, x\right) \bigvee(x) \bar{B}^{Q}\left(a, \alpha_{a}, x\right)\right\}$, and hence $P(a) \equiv(x) A^{Q}\left(a, \alpha_{a}, x\right)$.

So far this is for any $P(a)$ expressible in both 1-quantifier forms relative to $Q$. Now say in particular $P(a) \equiv(x)(E y) T_{2}^{Q}(a, a, x, y)$, which by Corollary Theorem 5 is so expressible. Then were there an $\alpha_{1}$ recursive in $Q$, since $\alpha_{a}=\lambda t \alpha_{1}\left(2^{a} \cdot 3^{t}\right)$, by $1.3 P(a) \equiv(x) A^{Q}\left(a, \alpha_{a}, x\right)$ would express $P(a)$ in a 1 -number-quantifier form relative to $Q$, contradicting that $(x)(E y) T_{2}^{Q}(a, a, x, y)$ is not so expressible (by the proof of IM Theorem V* Part II pp. 283, 292).

For $k=0,(x) R^{Q}(\alpha, x)$ can be developed into the form $(E \beta)(x) S(\alpha, \beta, x)$ with $S$ recursive (by Theorem 5), after which $(E \alpha)(E \beta)$ contract by (1) so that $(E \alpha)(x) R^{Q}(\alpha, x)$ assumes the form $(E \alpha)(x) R(\alpha, x)$ with $R$ recursive. If $\alpha$ makes $(x) R(\alpha, x)$ true, then (cf. (1)) $\lambda t(\alpha(t))_{0}$ would be an $\alpha_{1}$, so no such $\alpha$ can be recursive in $Q$.

Similarly for $k=1,2,3, \cdots,(E \alpha)(x) R^{Q}(\alpha, x)$ assumes a $k+1$-quantifier form $(E \alpha) B(\alpha)$ which is fulfillable by no $\alpha$ recursive in $Q$.

Corollary. For a suitable recursive $R$, the proposition $(E \alpha)(x) R(\alpha, x)$ is true, but $(x) R(\alpha, x)$ is false for every arithmetical function $\alpha(k=0)$.

For a suitable $k$-quantifier predicate $B(\alpha)$ with generality first, the proposition $(E \alpha) B(\alpha)$ is true, but $B(\alpha)$ is false for every function $\alpha$ arithmetical in $k$-quantifier predicates $(k=1,2,3, \cdots)$.

Proof. From the theorem, by taking as the $Q$ the $P$ of Theorem 6; or 
similarly to the theorem (and a bit more simply) by taking as the $P$ and $Q$ those for Theorem 6.

5.5 Using terminology suggested by Kreisel( $\left.{ }^{17}\right)$, say a class $C$ of functions is a basis for a class $D$ of predicates of a function variable, if for every predicate $B(\alpha)$ of $D,(E \alpha) B(\alpha) \equiv(E \alpha)[\alpha \in C \& B(\alpha)]$. We may allow $\alpha$ to range over arbitrary functions or bounded functions. We may let $D$ be the predicates expressible in a given form.

For the form $(E x) R(\alpha, x)$ ( $R$ recursive) with $\alpha$ arbitrary, (1) the functions vanishing except for a finite number of arguments, a fortiori the primitive recursive functions, are a basis (by IM Theorem IV* (6) pp. 281, 291, 292; or Kreisel [13]).

For $(x) R(\alpha, x)$ ( $R$ recursive) with $\alpha$ bounded, (2) the general recursive functions are not a basis, even when the bound is 1 (Kleene [7, §3]); but (3) the functions general recursive in 1-number-quantifier predicates are, even when $\alpha$ is merely bounded by a general recursive function of its argument (Kreisel [14, Note III]).

For $(x) R(\alpha, x)$ ( $R$ recursive) with $\alpha$ arbitrary, (4) the arithmetical functions are not a basis (Corollary Theorem 7), nor are much wider classes of functions (Theorem 7 with Theorem 9), including those definable in the extension of arithmetic of Wang [25] and Myhill [17] (Theorem 8); but (5) the functions general recursive in 1 -function-quantifier predicates are (since $(E \alpha)(x) R(\alpha, x) \rightarrow(x) R\left(\alpha_{0}, x\right)$, when we write $(x) R(\alpha, x) \equiv(x) R(\bar{\alpha}(x))$ using IM Theorem IV* with Footnote 2 here, and define $\alpha_{0}$ from $(E \beta)(x) R(u * \bar{\beta}(x))$ by the course-of-values recursion $\left.\alpha_{0}(a)=\mu y(E \beta)(x) R\left(\left(\bar{\alpha}_{0}(a) * 2^{y+1}\right) * \bar{\beta}(x)\right)\right)$. These results suggest asking whether the functions general recursive in predicates expressible in both 1-quantifier forms are a basis; a negative answer will be given in [10].

The results for $(x) R(\alpha, x)$ ( $R$ recursive) with $\alpha$ arbitrary extend to $(x)(E y) R(\alpha, x, y)$ ( $R$ recursive) with $\alpha$ bounded and to $A(\alpha)$ ( $A$ arithmetical) with $\alpha$ arbitrary or bounded, by the constructions of functions underlying the alterations of quantifiers by which the forms $(E \alpha)(x) R(\alpha, x),\left(E \alpha^{*}\right)(x)(E y)$ $R\left(\alpha^{*}, x, y\right),(E \alpha) A(\alpha)$ and $\left(E \alpha^{*}\right) A\left(\alpha^{*}\right)$ are equivalent $(3.7$ and 3.8 with $\left.3.1-3.6\left({ }^{10}\right)\right)$, even when for (4) the bound is 1 and for (5) any arithmetical function $\left({ }^{18}\right)$.

6. A hierarchy of predicates corresponding to the notations for constructive ordinals. $6.1 \mathrm{In}$ the system $S_{3}$ of notation for constructive ordinals of the first and second number classes $[4 ; 6 ; 9]$, a class $O$ of numbers and a relation $<_{o}$ between numbers are defined. The members $y$ of $O$ correspond to ordinals $|y|$ constituting a segment of the Cantor first and second number

(17) In correspondence; subsequently [14] appeared, in which Kreisel distinguishes prooftheoretically between "strong" and "weak" bases (p. 120).

(18) Indeed $A(\alpha)$, or the representing predicate of the bound for (5), can be of the $(E \beta)(x)$ form. 
classes determined by an ordinal $\omega_{1}$ (the "least nonconstructive ordinal"), and are partially ordered by $<_{0}$ so that $u<_{o} y$ implies $|u|<|y|$. (Indeed, $u<o y$ implies $u, y \in O$, by (IV) of $[6, \S 12]$ or $[9, \S 20]$.) If $y \in O$, then either $y=1$ and $|y|=0$, or $y=2^{z}$ with $z \neq 0$ and $|y|=|z|+1$, or $y=3 \cdot 5^{z}$ and $|y|=\lim \left|z_{n}\right|$, where $z_{n}=\{z\}\left(n_{o}\right), 0_{o}=1,(n+1)_{o}=2^{n_{o}},\{z\}(a) \simeq U\left(\mu y T_{1}(z, a, y)\right)$.

6.2 We define a predicate $H_{y}(a)$, for each $y$ which $\in O$, thus $\left({ }^{19}\right): H_{1}(a)$ $\equiv a=a$. If $2^{z} \in O$ and $z \neq 0$, then $H_{2^{z}}(a) \equiv(E x) T_{1}^{H_{z}}(a, a, x)$. If $3 \cdot 5^{z} \in O$, then $H_{3 \cdot 5^{z}}(a) \equiv H_{z_{(a)}}\left((a)_{0}\right)$.

6.3 In the remainder of this section we shall develop some elementary properties of the predicates $H_{y}$ (and the predicates $H_{y}^{Q} 6.5$ ).

Lemma 1. Given a predicate $R^{\Psi}\left(a_{1}, \cdots, a_{n}, x\right)$ partial recursive uniformly in $l$ functions or predicates $\Psi$ of $m_{1}, \cdots, m_{l}$ variables respectively, there is a primitive recursive function $\phi\left(a_{1}, \cdots, a_{n}\right)$ such that

$$
(E x) R^{\Psi}\left(a_{1}, \cdots, a_{n}, x\right) \equiv(E x) T_{1}^{\Psi}\left(\phi\left(a_{1}, \cdots, a_{n}\right), \phi\left(a_{1}, \cdots, a_{n}\right), x\right)
$$

for values of $\Psi, a_{1}, \cdots, a_{n}$ for which $\lambda x R^{\Psi}\left(a_{1}, \cdots, a_{n}, x\right)$ is completely defined.

Proof. Let $e$ be a uniform Gödel number of $\lambda a_{1} \cdots a_{n} t \mu x R^{\Psi}\left(a_{1}, \cdots, a_{n}, x\right)$ from $\Psi$, and let $\phi\left(a_{1}, \cdots, a_{n}\right)=S_{1}^{n, m_{1}}, \cdots m_{l}\left(e, a_{1}, \cdots, a_{n}\right)$ (cf. IM p. 343 Example 2).

Lemma 2. There is a partial recursive function $\iota(u, z)$ such that, if $u<_{o} 3 \cdot 5^{z}$, then $u \leqq o z_{\imath(u, z)}$.

Proof. By (VI) of $[6, \S 12]$ or $[9, \S 20],(E n)\left[u \leqq o z_{n}\right]$. By $[9, \S 21]$, there is a primitive recursive function $\operatorname{enm}(s, t)$ such that $\operatorname{enm}(1, t)=1$ and, if $s>o 1$, then $\operatorname{enm}(s, 0), \operatorname{enm}(s, 1), \operatorname{enm}(s, 2), \cdots$ is an enumeration of the numbers $<_{o s}$. So let $\iota(u, z) \simeq\left(\mu t\left[\operatorname{enm}\left(z_{(t) o},(t)_{1}\right)=u\right]\right)_{0}$. $=L_{i}^{\lambda_{a a}^{(19)} a}$.

(19) In the notation of Kleene-Post [11], $H_{2^{z}}=\left(H_{8}\right)^{\prime}, H_{3 \cdot 5^{2}}=H_{s_{0}} \cup H_{s_{1}} \cup H_{z_{2}} \cup \ldots, H_{i_{O}}$

This or an essentially equivalent correlation of predicates to the constructive ordinals has been treated by Mostowski [16] and Davis $[2 ; 3]$, and was known independently to Post and to the author. Mostowski's formulation stems quite directly from [5] and [15], utilizing analogies with the theory of projective sets; while the extensions discussed by Davis and here are constructed differently, using the idea of relative recursiveness. In $\$ 9$ below we obtain a matching between Mostowski's and the present extensions. Following Post $[19 ; 20]$ (also cf. [11]), let a predicate $A$ be of the same degree as (lower degree than) a predicate $B$, if $A$ is recursive in $B$ and also (but not) vice versa. Recursive predicates are of the degree 0 . Davis established the uniqueness of the degrees of the predicates $H_{y}$ associated with a given ordinal $|y|<\omega^{2}$, and showed the expressibility of $H_{v}$ for $|y|<\omega^{2}$ in the second-order predicate calculus with number variables as the lower type; Mostowski $[16,4.5]$ stated the latter (for his extension) for $|y|<\omega_{1}$. The present $\$ 8$ with 3.8 , besides providing a simple proof of the latter (for $H_{y}$, and via $\$ 9$ for Mostowski's extension) for $|y|<\omega_{1}$, shows that only one set-quantifier needs to be used, which can be of either kind. In [22] Spector will extend the uniqueness to all $|y|<\omega_{1}$. 
Lemma 3. There are functions $\rho_{1}(t, u, y, a), \rho_{2}(u, y)$ and $\rho(u, y)$, primitive, partial and primitive recursive, respectively, such that (writing $\rho(u, y, a)$ $\left.\simeq \rho_{1}\left(\rho_{2}(u, y), u, y, a\right)\right)$, if $y \in O$ and $u \leqq o y$, then

$$
H_{u}(a) \equiv H_{y}(\rho(u, y, a))
$$

and $H_{u}$ is primitive recursive in $H_{y}$ with Gödel number $\rho(u, y)$.

Proof. The proof that (12) holds for $y \in O$ and $u \leqq o y$ will be by induction on $y$, for a fixed $u$. Instead of defining $\rho_{1}$ and $\rho_{2}$ first, we shall begin with the cases for the induction (cf. (I), (II) and (V) of $[6, \S 12]$ or $[9, \S 20]$ ), in each case deriving properties of $\rho_{1}$ and $\rho_{2}$ sufficient to treat the case. Then in conclusion we shall verify that $\rho_{1}$ and $\rho_{2}$ can be defined to combine these properties. CASE 1: $y=u$ and $y \in O$. Then $H_{u}(a) \equiv H_{y}(a)$, so it will suffice to take $\rho_{1}(t, u, y, a)=a$ and $\rho_{2}(u, y)=0$. CASE 2: $y=2^{z}, z \in O$ (so $\left.z \neq 0\right)$ and $u \leqq o z$. Then $z=(y)_{0}$. By the hypothesis of the induction, $H_{u}(a) \equiv H_{z}\left(\rho_{1}\left(\rho_{2}(u, z)\right.\right.$, $u, z, a))$. But $H_{z}(c) \equiv(E x) H_{z}(c)$, and $H_{y}(a) \equiv(E x) T_{1}^{H_{z}}(a, a, x)$; so by Lemma 1 for $l=n=1, H_{z}(c) \equiv H_{y}(\phi(c))$ for a primitive recursive $\phi$. Thus $H_{u}(a)$ $\equiv H_{y}\left(\phi\left(\rho_{1}\left(\rho_{2}(u, z), u, z, a\right)\right)\right)$. So it will suffice to take $\rho_{1}(t, u, y, a)$ $=\phi\left(\rho_{1}\left((t)_{0}, u,(y)_{0}, a\right)\right)$ for $t \neq 0$, and $\rho_{2}(u, y)=2^{\rho_{2}\left(u,(y)_{0}\right)}$. CASE $3: y=3 \cdot 5^{z}$ and $u<o y$. Then $z=(y)_{2}$. By Lemma 2, the hypothesis of the induction, and the definition of $H_{y}$,

$$
\begin{aligned}
H_{u}(a) & \equiv H_{z_{\iota}(u, z)}\left(\rho_{1}\left(\rho_{2}\left(u, z_{\iota(u, z)}\right), u, z_{\iota}(u, z), a\right)\right) \\
& \left.\equiv H_{y}\left(2^{\rho_{1}\left(\rho_{2}\left(u, z_{\iota}(u, s)\right), u, z_{\iota}(u, s)\right.}, a\right) \cdot 3^{\imath(u, z)}\right) .
\end{aligned}
$$

So it will suffice to take $\rho_{1}(t, u, y, a)=2^{\rho_{1}\left((t)_{0}, u,(t)_{1}, a\right)} \cdot 3^{(t) 2}$ for $t \neq 0$, and $\rho_{2}(u, y)=2^{\rho_{2}\left(u,\left[(y)_{2}\right]_{n}\right)} \cdot 3^{\left[(y)_{2}\right]_{n}} \cdot 5^{n}$ where (for abbreviation) $n=\iota\left(u,(y)_{2}\right)$. CoNCLUSION. Combining the cases, it suffices for $\rho_{1}$ to take

$$
\rho_{1}(t, u, y, a)= \begin{cases}\phi\left(\rho_{1}\left((t)_{0}, u,(y)_{0}, a\right) j\right. & \text { if } y=2^{(y)_{0}} \neq u \& t \neq 0, \\ 2^{\rho_{1}\left((t)_{0}, u,(t)_{1}, a\right)} \cdot 3^{(t)_{2}} & \text { if } y=3^{(y)_{2}} \neq u \& t \neq 0, \\ a \text { otherwise. } & \end{cases}
$$

This is a nested course-of-values recursion, so it defines a primitive recursive $\rho_{1}$, by Péter $\left[18, \S 2\right.$ especially 20]. It suffices for $\rho_{2}$ to take $\rho_{2}(u, y) \simeq\{r\}(u, y)$ where $r$ is a solution for $w$ of the equation

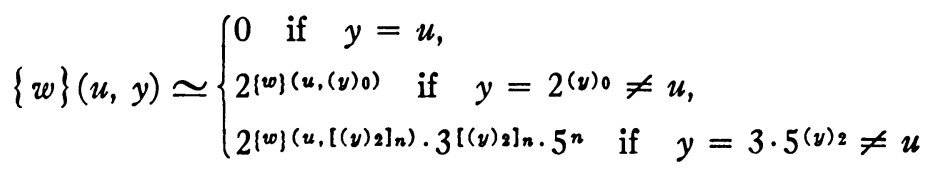

$\left(n=\imath\left(u,(y)_{2}\right)\right)$; the definition by cases in this equation is to be understood in the strong sense IM Theorem XX (c) p. 337. A solution $r$ is given by the recursion theorem (IM Theorem XXVII p. 352). Finally, let $\rho(u, y)$ 
$=S_{1}^{2,1}(h, u, y)$ where $h$ is a uniform Gödel number of $\lambda u y a A(\rho(u, y, a))$ from an unspecified predicate $A$.

A primitive recursive function $t_{o}$ is defined in $[9, \S 22]$ so that, for $u, y \in O, u+o y \in O$ and $|u+o y|=|u|+|y|$. Then $u \leqq o u+o y$ but not always $y \leqq o u+o y[9, \S 22$ (XIX) and Footnote 30].

Lemma 4. There are functions $\sigma(u, y, a)$ and $\sigma(u, y)$, partial and primitive recursive, respectively, such that, if $u, y \in O$, then

$$
H_{y}(a) \equiv H_{u+o v}(\sigma(u, y, a))
$$

and $H_{y}$ is general recursive in $H_{u+o y}$ with Gödel number $\sigma(u, y)$.

Proof, by induction on $y$. CASE $1: y=1$. By the definition in $[9, \S 22]$, $u+o y=u \geqq o 1=y$. So by Lemma $3, H_{y}(a) \equiv H_{u+o y}(\rho(1, u, a))$. CASE 2: $y=2^{z}$ and $z \in O$. By definition, $u+o y=2^{u+o z}$. By the definitions of $H_{y}$ and $H_{u+o y}$, the hypothesis of the induction, and Lemma $1, H_{y}(a) \equiv H_{u+o y}(\phi(s, u, z, a))$, where $s$ is a Gödel number of $\sigma$, and $\phi$ is primitive recursive. CASE 3: $y=3 \cdot 5^{z}$ and $y \in O$. By def., $u+o y=3 \cdot 5^{v}$ where $v_{n}=u+o z_{n}$. By the def. of $H_{y}$, the hyp. ind., and the def. of $H_{u+o y}, H_{y}(a) \equiv H_{u+o y}\left(\left[2 \exp \sigma\left(u, z_{(a)_{1}},(a)_{0}\right)\right] \cdot 3^{(a)_{1}}\right)$. Conclusion. Take $\sigma(u, y, a) \simeq\{s\}(u, y, a)$ where $s$ is a solution for $w$ of the equation

$$
\{w\}(u, y, a) \simeq\left\{\begin{array}{l}
\rho(1, u, a) \text { if } y=1, \\
\phi\left(w, u,(y)_{0}, a\right) \text { if } y=2^{(y)_{0}} \neq 1, \\
{\left[2 \exp \{w\}\left(u,\left[(y)_{2}\right]_{(a)_{1}},(a)_{0}\right)\right] \cdot 3^{(a)_{1}} \text { if } y=3 \cdot 5^{(y)_{2}} .}
\end{array}\right.
$$

6.4 Let $Q$ be say a 1-place predicate. In the definitions of $O,<_{o}, \|, \omega_{1},+_{o}$, let recursiveness in $Q$ take over the role of recursiveness. This leads to a system of notations for ordinals constructive in $Q$, with respective notions $O^{Q},<\&, \| Q, \omega_{1}^{Q},+_{o}^{1}[9, \S 30]\left({ }^{20}\right)$.

It is not hard to see that a given number $y$ may $\in O$, but not to $O^{Q}$, or vice versa; or to $O^{A}$ but not to $O^{B}$. On the other hand, a number $y$ may belong to $O$, to $O^{A}$ and to $O^{B}$, and represent different ordinals $|y|,|y|^{A}$ and $|y|^{B}$ in the respective systems.

In each system $O$ or $O^{Q}$, proceeding upward in terms of the ordering $<o$ or $<8$, at each limit ordinal $\alpha<\omega_{1}$ or $<\omega_{1}^{Q}$ there is a branching with $\aleph_{0}$ branches, due to the possibility of choosing between different ascending sequences $\left|z_{n}\right|$ or $\left|z_{n}^{Q}\right| \mathcal{Q}$ to approach the limit ordinal, between different functions partial recursive absolutely or in $Q$ to take the values $z_{n}$ or $z_{n}^{Q}$ for the arguments $n_{o}$, and between different Gödel numbers absolutely or from $Q$ for those functions. We call notations $y$ and $\bar{y}$ of the same or different systems homologous, if they differ only by choices of the second and third kinds. We write

(20) We write ${ }_{{ }_{0}^{1}}^{1}$ instead of $+_{o}^{0}$ because only the number 1 of the arguments of $Q$ matters [9, Footnote 44]. Similar superscripts on $\pi, \pi_{1}, \pi, \nu, \kappa(\operatorname{Lemmas} 5,6,8)$ are omitted here. 
$(y, A) \sim(\bar{y}, B)$ to say that $y$ as notation of $O^{A}$ is homologous to $\bar{y}$ as notation of $O^{B}$. In detail, $(y, A) \sim(\bar{y}, B)$ can be defined for $y \in O^{A}$ by induction on $y$ thus: 1 . $(1, A) \sim(\bar{y}, B)$ if $\bar{y}=1$. 2. For $2^{z} \in O^{A}$ and $z \neq 0,\left(2^{z}, A\right) \sim(\bar{y}, B)$ if $\bar{y}=2^{z}$ where $(z, A) \sim(\bar{z}, B)$. 3. For $3 \cdot 5^{z} \in O^{A},\left(3 \cdot 5^{z}, A\right) \sim(\bar{y}, B)$ if $\bar{y} \in O^{B}$ and $\bar{y}=3 \cdot 5^{\bar{z}}$ where $\left(z_{n}^{A}, A\right) \sim\left(\bar{z}_{n}^{B}, B\right)$ for all $n$. For $y \notin O^{A},(y, A) \sim(\bar{y}, B)$ shall be false. Now $(y, A) \sim(\bar{y}, B)$ implies that $y \in O^{A}, \bar{y} \in O^{B}$ and $|y|^{A}=|\bar{y}|^{B}$. Similarly we write $(y, A) \sim(\bar{y})$ to say that $y$ as notation of $O^{A}$ is homologous to $\bar{y}$ as notation of $O$; etc. The homology relation $\sim$ is symmetric, reflexive and transitive. We say homologous notations possess the same $h$-type.

Lemma 5. There is a primitive recursive function $\pi(e, y)$ such that, if $y \in O^{A}$ and $A$ is recursive in $B$ with Gödel number $e$, then $(y, A) \sim(\pi(e, y), B)$. Moreover, $u<{ }_{0}^{A} y \rightarrow \pi(e, u){ }_{0}^{B} \pi(e, y)$.

There is a primitive recursive function $\pi_{1}(e, y)$ such that, if $y \in O^{A}$ and $A$ is recursive with Gödel number e, then $(y, A) \sim\left(\pi_{1}(e, y)\right)$. Moreover, $u<{ }_{0}^{A} y$ $\rightarrow \pi_{1}(e, u)<_{o} \pi_{1}(e, y)$.

There is a primitive recursive function $\pi(y)$ such that, if $y \in O$, then $(y) \sim(\pi(y), B)$. Moreover, $u<o y \rightarrow \pi(u)<{ }_{0}^{B} \pi(y)$.

Proof, for $\pi(e, y)$, by induction on $y$. In Case $1, \pi(e, y)=1$. In Case 2, $\pi(e, y)=2^{\pi(e, z)}$. In Case $3, \pi(e, y)=3 \cdot 5^{z}$ where $\bar{z}_{n}^{B}=\pi\left(e, z_{n}^{A}\right)$. Elaborating and combining the cases, we take $\pi(e, y) \simeq\{p\}(e, y)$ where $p$ is a solution for $w$ of the equation

$$
\{w\}(e, y) \simeq\left\{\begin{array}{l}
1 \quad \text { if } y=1 \\
2^{\{w\}\left(e,(y)_{0}\right)} \text { if } y=2^{(y)_{0}} \neq 1 \\
3 \cdot 5^{S_{1}{ }^{3,1}\left(h_{3}, w, e,(y)_{2}\right)} \text { if } y=3 \cdot 5^{(y) 2}, \\
0 \text { otherwise, }
\end{array}\right.
$$

where $h_{3}$ is a uniform Gödel number of $\lambda$ peza $\{p\}\left(e,\{z\}^{\lambda c \mid e\}^{B}(c)}(a)\right)$ from $B$. This gives $\pi$ as a partial recursive function. But on replacing in the equation " $\{w\}$ " by " $\pi$ " and the remaining " $w$ " by " $p$ ", we have a course-of-values recursion for $\pi(e, y)$, so $\pi$ is primitive recursive (Péter $[18, \S 1]$, IM $\S 46$ ).

Leмma 6. There is a primitive recursive function $\nu(a)$ such that

$$
Q(a) \equiv \nu(a) \in O^{Q} .
$$

So $Q$ is primitive recursive uniformly in $\lambda a a \in O^{Q}$.

Proof. Let $h$ be a uniform Gödel number from $Q$ of the function

$$
\phi(a, x)=\left\{\begin{array}{l}
x \text { if } x \neq a_{o}, \\
a_{O} \text { if } x=a_{O} \& Q(a), \\
0 \text { if } x=a_{O} \& \bar{Q}(a),
\end{array}\right.
$$

and let $\nu(a)=3 \cdot 5^{S^{1,1}(h, a)}$. 
As superscript, $O$ shall mean $\lambda a a \in O$, and $O^{Q}$ shall mean $\lambda a a \in O^{Q}$.

Lemma 7. There is a number $w_{1}$ such that $w_{1} \in O^{\circ}$ and $\left|w_{1}\right|^{O}=\omega_{1}$. There is a number $w_{1}^{1}$ such that $w_{1}^{1} \in O^{O^{Q}}$ and $\left|w_{1}^{1}\right|^{Q}=\omega_{1}^{Q}$.

Proof, for $w_{1}$. Let $w_{1}=3 \cdot 5^{z}$, where $z$ is a Gödel number of $\lambda t \phi$ (nat $\left.(t)\right)$ from $\lambda a \quad a \in O$, nat $(t)=\mu n_{n<t} t=n_{O}$ (so nat $\left(n_{O}\right)=n$ ), and

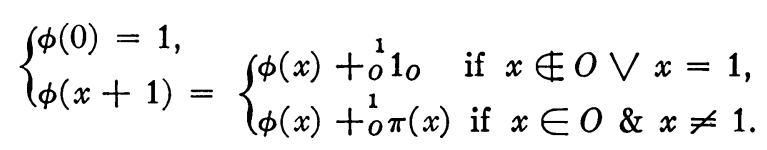

Suppose $A$ is recursive, and $B$ is recursive in $C$. By Lemma $5, \omega_{1}=\omega_{1}^{A}$ $\leqq \omega_{1}^{B} \leqq \omega_{1}^{C}$. By Lemma $7, \omega_{1}<\omega_{1}^{0}<\omega_{1}^{0^{0}}<\cdots$.

6.5 We define $H_{y}^{Q}(a)$, for each $y \in O^{Q}$, thus: $H_{1}^{Q}(a) \equiv Q(a)$. If $2^{z} \in O^{Q}$ and $z \neq 0$, then $H_{2^{z}}^{Q}(a) \equiv(E x) T_{1}^{Q, H_{z}{ }^{Q}}(a, a, x)$. If $3 \cdot 5^{z} \in O^{Q}$, then

$$
H_{3 \cdot 5^{z}}^{Q}(a) \equiv H_{z_{(a)_{1}}^{Q}}^{Q}\left((a)_{0}\right) \text {. }
$$

Clearly $H_{y}$ depends only on the h-type of $y$ as a notation of $O$, and $H_{v}^{Q}$ only on $Q$ and the h-type of $y$ as a notation of $O^{Q}$.

6.6 By relativization to $Q$ in the proofs of Lemmas $2-4$ using $O^{Q}, H_{y}^{Q}$, etc. instead of $O, H_{y}$, etc. we obtain Lemmas $2^{*}-4^{*}$ with functions $\iota^{Q}(u, z)$, $\rho_{1}^{1}(t, u, y, a), \rho_{2}^{Q}(u, y), \rho^{1}(u, y), \rho^{Q}(u, y, a), \sigma^{Q}(u, y, a), \sigma^{1}(u, y)$ taking over the role of the former functions without superscripts (those with superscript "1" are primitive recursive, those with superscript "Q" partial recursive, uniformly in $Q) ; \rho^{1}(u, y)$ is a Gödel number of $H_{u}^{Q}$ from $Q, H_{y}^{Q}$, and $\sigma^{1}(u, y)$ of $H_{y}^{Q}$ from $Q, H_{u+0^{1} y}^{Q}$. As an additional clause of

Lemma $3^{*}$. There is a function $\rho^{Q}(u, y)$ partial recursive uniformly in $Q$ such that, if $y \in O^{Q}$ and $u \leqq{ }^{Q} y$, then $H_{u}^{Q}$ is primitive recursive in $H_{v}^{Q}$ with Gödel number $\rho^{Q}(u, y)$. So, if $y \in O^{Q}$, then $Q$ is recursive in $H_{y}^{Q}$ with Gödel number $\rho^{Q}(1, y)$.

For let $\rho^{Q}(u, y)=S_{1}^{3,1}\left(h, \rho_{2}^{Q}(u, y), u, y\right)$ where $h$ is a uniform Gödel number of $\lambda$ tuya $A\left(\rho_{1}^{1}(t, u, y, a)\right)$ from $A$.

6.7 Lemma 8. There is a primitive recursive function $\kappa(e, y)$ such that, if $A$ is recursive in $B$ with Gödel number $e$ and $(y, A) \sim(\bar{y}, B)$, then $H_{\nu}^{A}$ is recursive in $A, H_{y}^{B}$ with Gödel number $\kappa(e, y)$.

Proof, by induction on $y$. In Case $2, H_{y}^{A}(a) \equiv H_{y}^{B}(\phi(k, e, z, a))$, where $k$ is a Gödel number of $\kappa$, and $\phi$ is primitive recursive. So take $\kappa(e, y)$ $=S_{1}^{3,1,1}\left(h_{2}, k, e,(y)_{0}\right)$ for $h_{2}$ a uniform Gödel number of $\lambda k e z a P(\phi(k, e, z, a))$ from $A, P$. In Case $3, H_{\nu}^{A}(a) \equiv R^{A, H_{y}^{B}}(k, e, z, a)$ where $R^{A, P}(w, e, z, a)$ $\cong\left\{\{w\}\left(e, z_{(a)_{1}}^{A}\right)\right\}^{A, \lambda_{C} P\left(2^{c} \cdot\left[3 \exp (a)_{1}\right]\right)}\left((a)_{0}\right)=0$.

6.8 We included the (first) $Q$ of $T_{1}^{Q, H_{z}^{Q}}$ in the definition of $H_{2^{z}}^{Q}$ because 
discussions relative to a fixed $Q$ tend to be easier when for each recursivity in $\Psi$ the $\Psi$ include $Q$. However that $Q$ is redundant. To prove this, let $\dot{H}_{\nu}^{Q}(a)$ for $y \in O^{Q}$ be defined like $H_{y}^{Q}(a)$ except that $\dot{H}_{2^{2}}^{Q}(a) \equiv(E x) T_{1}^{\dot{H}_{z}{ }^{Q}}(a, a, x)$ simply. A Lemma $\dot{3}^{*}$ holds like $3^{*}$ with $\dot{H}_{y}^{Q}, \dot{\rho}_{1}^{1}, \dot{\rho}_{2}^{Q}$, etc. in place of $H_{y}^{Q}, \rho_{1}^{1}, \rho_{2}^{Q}$, etc. (and $\dot{\rho}_{1}^{1}, \dot{\rho}_{2}^{Q}$ can be the $\rho_{1}, \rho_{2}^{Q}$ of the proofs of Lemmas 3 and $3^{*}$ ).

LemMA 9. There is a function $\lambda^{Q}(y, a)$ partial recursive uniformly in $Q$ such that, if $y \in O^{Q}$, then

$$
H_{y}^{Q}(a) \equiv \dot{H}_{y}^{Q}\left(\lambda^{Q}(y, a)\right) .
$$

Proof, by induction on $y$. In Case 2, using the def. of $H_{y}^{Q}$, the hyp. ind., and Lemma $\dot{3}^{*}, H_{y}^{Q}(a) \equiv(E x) R^{H_{z}^{Q}}\left(l, \dot{\rho}^{Q}(1, z), z, a, x\right)$, where $l$ is a uniform Gödel number of $\lambda^{Q}$ from $Q$, and $R^{A}$ is partial recursive uniformly in $A$. So by the def. of $\dot{H}_{\nu}^{Q}$ and Lemma $1, H_{\nu}^{Q}(a) \equiv \dot{H}_{\nu}^{Q}\left(\phi\left(l, \dot{\rho}^{Q}\left(1,(y)_{0}\right),(y)_{0}, a\right)\right)$ for a primitive recursive $\phi$.

LeMma 10. There is a function $\mu^{Q}(y, a)$ partial recursive uniformly in $Q$ such that, if $y \in O^{Q}$, then

$$
\dot{H}_{y}^{Q}(a) \equiv H_{y}^{Q}\left(\mu^{Q}(y, a)\right) .
$$

6.9 Applying Lemma 8 with Lemma $3^{*}$, for $A, B$ first as $Q_{1}, Q_{2}$ and then as $Q_{2}, Q_{1}$, when $Q_{1}, Q_{2}$ are of the same degree( $\left.{ }^{19}\right)$, we have that the degree of $H_{y}^{Q}$ depends only on the degree $\mathbf{q}$ of $Q$ and the h-type of $y$ as a notation. of $O^{Q}$. We write this degree as $\mathbf{q}_{(y, Q)}$, or $\mathbf{q}_{h}$ where $h \sim(y, Q)$, or sometimes even $\mathbf{q}^{(\alpha)}$ where $\alpha=|y|^{Q\left({ }^{19}\right)}$. Then by Lemmas $3^{*}$ and 8 ,

$$
y \in O^{A} \& \mathrm{a} \leqq \mathrm{b} \rightarrow \mathrm{a} \leqq \mathrm{a}_{(y, A)} \leqq \mathrm{b}_{(y, A)}
$$

for $\mathbf{a}$ and $\mathbf{b}$ the degrees of $A$ and $B$. The degree of $H_{y}$ is then $\mathbf{0}_{(y)}$ (or $\mathbf{0}_{h}$ for $h \sim(y)$, or $0^{(\alpha)}$ for $\alpha=|y|$ ), since by Lemma $5 H_{y}(a) \equiv \dot{H}_{\boldsymbol{x}(y)}^{\lambda a} a(a)$, and by Lemmas 9 and 10 with $\dot{3}^{*}$ and $3^{*} \dot{H}_{\boldsymbol{\pi}(y)}^{\lambda a=a}$ and $H_{\boldsymbol{\pi}(y)}^{\lambda a=a}$ have the same degree. By Lemmas $9,10, \dot{3}^{*}$ and $3^{*}$, our present notations $\mathbf{q}^{(\alpha)}$ are consistent with those of Kleene and Post [11, 1.4 and 4.1].

By Lemma $3^{*}$, if $y \in O^{Q}$ and $u \leqq \delta y$, then $\mathbf{q}_{(u, Q)} \leqq \mathbf{q}_{(y, Q)}$. Using this with the inequality $\mathbf{a}<\mathbf{a}^{\prime}$ from $[11,1.4]$, we easily see that

$$
u<\stackrel{Q}{o} y \rightarrow \mathrm{q}_{(u, Q)}<\mathrm{q}_{(y, Q)} .
$$

The predicate $H_{w_{1}}^{0}$ (cf. Lemma 7) is of higher degree than $H_{y}^{0}$ for any $y$ with $(y, \lambda a a \in O) \sim(x)$, as follows from Lemmas $4^{*}$ and $3^{*}$ with (18).

7. Wang's and Myhill's extensions of arithmetic. It was first observed by Kalmár (cf. Skolem [21]) that nonarithmetical predicates are definable by employing induction over the natural numbers so that quantifiers enter in the induction step. Recently Wang [25] and Myhill [17] independently of each other formulated general schemes of definition by induction under 
which the examples of such definitions in the literature fall. Their investigations concern questions of provability in formalisms, which we do not enter into here.

THEOREM 8. Wang's predicates indirectly definable by induction (relative to number theory), and likewise Myhill's predicates definable by his rule of creative definition $\mathrm{RD}$ used together with $0,+1$, explicit definition and the leastnumber operator, are each exactly the predicates of degrees $\leqq 0^{(\nu)}$ for $\nu<\omega^{2}$. Wang's predicates definable by induction (relative to number theory) are exactly those of degrees $\leqq 0^{(\omega)}$.

Proof, from Lemma 11. Wang's schema (D) is superficially more general than Myhill's RD in that the induction is of the course-of-values type, but we shall see that this feature is eliminable in the usual way (Péter [18], IM \$46).

Call a predicate definable by 0 (repeated) applications of (D), if it is recursive; by $r+1$ applications, if it comes by (D) from predicates definable by $\leqq r$ applications (including one definable by $r$ applications).

Wang's predicates indirectly definable by induction (relative to number theory) are then the predicates definable by $r$ applications of (D) for some $r$, or equivalently the predicates arithmetical in predicates definable by $\leqq r$ applications of (D) for some $r\left({ }^{21}\right)$.

Now we prove by induction on $r$ that a predicate definable by $r$ applications of (D) is of degree $\leqq 0^{(r \omega)}$ (and hence a predicate arithmetical in predicates definable by $\leqq r$ applications of $(\mathrm{D})$ is of degree $\leqq 0^{(r \omega+s)}$ for some s).

For the induction step, let $P(a, k)$ be defined by an $r+1$-st application of (D). The predicates defined by $\leqq r$ applications which enter into its definition are of degrees $\leqq 0^{(r \omega)}$, by the hypothesis of the induction; so they are all recursive in a particular predicate $A(a)$ of the degree $0^{(r \omega)}$. Let $\pi(k, a)$ be the representing function of $P(a, k)$, and let $\phi(a, k)=\tilde{\pi}(k ; a)$. Using familiar methods $(2.4,2.5$, IM §46), we can see that $\phi$ satisfies the hypotheses of the following lemma (indeed for $p=0$ ). Therefore $\phi$ and hence $P$ is of degree $\leqq$ the degree $a^{(\omega)}$ of $L^{A}[11,4.1]$. But $a=0^{(r \omega)}$, so $a^{(\omega)}$ $=0^{((r+1) \omega)}$.

Of course $r \omega<\omega^{2}$. That all predicates of degree $\leqq 0^{(v)}$ for $\nu<\omega^{2}$ are de-

(21) The second formulation may permit an $r$ smaller by one. Wang's formulation is the first but with $r \geqq 2$ and " $=r$ " instead of " $\leqq r$ " in the preceding paragraph. These three formulations are equivalent, since arithmetical operations including the identical operation can be reformulated as applications of (D). Arithmetical operations preceding an application of (D) need not be considered, because they can be assimilated into the application of (D). It follows from the present treatment that each predicate indirectly definable by induction is definable by a linear series of $r$ applications of (D) (of especially simple form) used to define $\left.M^{1}, \cdots, M^{r(22}\right)$, followed by a recursive operation (or possibly decreasing $r$ by one, by an arithmetical operation). 
finable using Wang's (D) or Myhill's RD follows from the fact that the particular predicates $L^{0}, L^{1}, L^{2}, \cdots$, where $L^{0}(a, k) \equiv a=a$ and $L^{r+1}(a, k)$ $\equiv L^{\lambda a L^{r}\left((a)_{0},(a)_{1}\right)}(a, k)$ are so definable and have the respective degrees $0^{(0 \omega)}$, $0^{(1 \omega)}, 0^{(2 \omega)}, \cdots\left({ }^{22}\right)$. If $\nu<\omega^{2}$, then $\nu \leqq r \omega$ for some $r$, so using (18) every predicate of degree $\leqq 0^{(\nu)}$ for a $\nu<\omega^{2}$ is recursive in one of $L^{0}, L^{1}, L^{2}, \cdots$.

Lemma 11. If $\lambda a \phi(a, 0)$ is arithmetical in $A$, and $\lambda a k \phi(a, k+1)$ is arithmetical uniformly in $A, \lambda a \phi(a, k)$, then $\lambda a k \phi(a, k)$ is recursive in $L^{A}$.

The first hypothesis means that $\phi(a, 0)=w \equiv B(\alpha, a, w)$, the second that $\phi(a, k+1)=w \equiv C(\alpha, \lambda a \phi(a, k), a, k, w)$, where $B(\xi, a, w)$ and $C(\xi, \eta, a, k, w)$ are arithmetical, and $\alpha$ is the representing function of $A$.

Proof. Then $B(\xi, a, w)$ and $C(\xi, \eta, a, k, w)$ are expressible in forms of the arithmetical hierarchy 3.1 , say with $p$ and $q$ quantifiers respectively. We shall show that $\lambda a \phi(a, k)$ is recursive in $L_{q \mathbf{k}+p}^{A}$ recursively in $k[11,3.6$ and Footnote 30$]$.

Say e.g. (with $p=1, q=3) B(\xi, a, w) \equiv(E x) R(\xi, a, w, x)$ and $C(\xi, \eta, a, k, w)$ $\equiv(x)(E y)(z) S(\xi, \eta, a, k, w, x, y, z)$ with $R$ and $S$ recursive. Define $\psi$ by

$$
\begin{aligned}
\psi(a, 0) & =0, \\
\psi(a, 1) & =\mu w(E x) R(\alpha, a, w, x), \\
\psi(a, 3 k+2) & =\left\{\begin{array}{l}
0 \text { if }(z) S\left(\alpha, \lambda a \psi(a, 3 k+1),(a)_{0}, k,(a)_{1},(a)_{2},(a)_{3}, z\right), \\
1 \text { otherwise },
\end{array}\right. \\
\psi(a, 3 k+3) & =\left\{\begin{array}{l}
0 \text { if }(E y)\left[\psi\left(2^{(a)_{0}} \cdot 3^{(a)_{1}} \cdot 5^{(a)_{2}} \cdot 7^{y}, 3 k+2\right)=0\right], \\
1 \text { otherwise, }
\end{array}\right. \\
\psi(a, 3 k+4) & =\mu w(x)\left[\psi\left(2^{a} \cdot 3^{w} \cdot 5^{x}, 3 k+3\right)=0\right] .
\end{aligned}
$$

Then $\phi(a, k)=\psi(a, 3 k+1)$. On combining the cases of the definition of $\psi(a, k)$ for $k>0$, it comes under [11, 4.1, Lemma 3 with Footnote 30], so $\lambda a \psi(a, k)$ is recursive in $L_{\mathbf{k}}^{\boldsymbol{A}}$ recursively in $k$, say with Gödel number $\epsilon(k)$ (so $\lambda a \phi(a, k)$ is recursive in $L_{3 k+1}^{A}$ recursively in $k$, with Gödel number $\epsilon(3 k+1))$. A fortiori $[11,3.6], \psi$ and hence $\phi$ is recursive in $L^{A}$.

8. Expressibility of the predicates $H_{y}$ for $y \in O$ in both 1-quantifier forms. 8.1 If $P(a)$ is expressible in both 1-quantifier forms, then

$$
P(a) \equiv(\alpha)(E x) T_{1}^{\alpha}\left((e)_{0}, a, x\right) \equiv(E \alpha)(x) \bar{T}_{1}^{\alpha}\left((e)_{1}, a, x\right)
$$

when $e=2^{f} \cdot 3^{o}$ for the $f$ and $g$ of Theorem 2 (9) and (10). Any number $e$ for (19) we call an index of $P(a)$ as a predicate expressible in both 1-quantifier forms. Indices for dealing with predicates expressible in both 1-quantifier forms relative to $Q$, or in both $k+1$-quantifier forms absolutely or relatively, are defined similarly.

${ }^{(22)}$ Or similarly with $M$ (IM pp. 287, 292, [11, Footnote 29]). 
8.2 Lemma 12 generalizes Theorem 2 (9) and (10) for $l \geqq 0$ to include the case of parameters $b_{1}, \cdots, b_{n}$ and the case of a partial recursive $R$.

Lemma 12. Given a predicate $R^{\Psi}\left(b_{1}, \cdots, b_{n}, a, \alpha, x\right)$ partial recursive uniformly in l functions or predicates $\Psi$ of $m_{1}, \cdots, m_{l}$ variables respectively, there are primitive recursive functions $\phi$ and $\psi$ such that

$$
\begin{aligned}
& (\alpha)(E x) R^{\Psi}\left(b_{1}, \cdots, b_{n}, a, \alpha, x\right) \equiv(\alpha)(E x) T^{\Psi, \alpha}\left(\phi\left(b_{1}, \cdots, b_{n}\right), a, x\right), \\
& (E \alpha)(x) R^{\Psi}\left(b_{1}, \cdots, b_{n}, a, \alpha, x\right) \equiv(E \alpha)(x) \bar{T}^{\Psi, \alpha}\left(\psi\left(b_{1}, \cdots, b_{n}\right), a, x\right)
\end{aligned}
$$

for values of $\Psi, b_{1}, \cdots, b_{n}$, a for which $\lambda \alpha x R^{\Psi}\left(b_{1}, \cdots, b_{n}, a, \alpha, x\right)$ is completely defined. (Similarly for $n=0$, or with more function variables quantified in a given manner, or with more variables a.)

Proof. Let $e$ be a uniform Gödel number of $\lambda b_{1} \cdots b_{n} a \mu x R^{\Psi}\left(b_{1}, \cdots, b_{n}\right.$, $\alpha, x)$ from $\Psi, \alpha$, and let $\phi\left(b_{1}, \cdots, b_{n}\right)=S_{1}^{n}, m_{1}, \cdots, m_{l}, 1\left(e, b_{1}, \cdots, b_{n}\right)$.

8.3 THEOREM 9. There is a primitive recursive function $\tau(y)$ such that, if $y \in O$, then $\tau(y)$ is an index of $H_{y}(a)$ as a predicate expressible in both 1-quantifier forms.

There is a primitive recursive function $\tau^{1}(y)$ such that, if $y \in O^{Q}$, then $\tau^{1}(y)$ is an index of $H_{y}^{Q}(a)$ as a predicate expressible in both 1-quantifier forms relative to $Q$.

Proof, for $\tau$, by induction on $y$ (cf. the proofs of Lemmas 3 and 4). CASE 1 : $y=1$. Using Theorem 2, for a suitable number $f_{1}, H_{1}(a) \equiv a=a \equiv(\alpha)(E x) a=a$ $\equiv(\alpha)(E x) T_{1}^{\alpha}\left(f_{1}, a, x\right)$; and similarly $H_{1}(a) \equiv(E \alpha)(x) \bar{T}_{1}^{\alpha}\left(g_{1}, a, x\right)$. So it will suffice to take $\tau(y)=2^{f_{1}} \cdot 3^{y_{1}}$. CASE 2: $y=2^{z}$ and $z \in O$. By the hyp. ind., $H_{z}(a) \equiv(\alpha)(E x) T_{1}^{\alpha}\left((\tau(z))_{0}, \quad a, x\right) \equiv(E \alpha)(x) \bar{T}_{1}^{\alpha}\left((\tau(z))_{1}, \quad a, x\right)$; and by def., $H_{y}(a) \equiv(E x) T_{1}^{H_{z}}(a, a, x)$. First replacing $\tau(z)$ by a variable $u$ and applying Theorem 5, then changing $u$ to $\{t\}(z)$ where $t$ is a Gödel number of $\tau$, we obtain

$$
H_{y}(a) \equiv(\alpha)(E x) R_{2}(t, z, a, \alpha, x) \equiv(E \alpha)(x) S_{2}(t, z, a, \alpha, x)
$$

with $R_{2}$ and $S_{2}$ partial recursive, whence by Lemma 12 for $l=0, n=2$,

$$
H_{y}(a) \equiv(\alpha)(E x) T_{1}^{\alpha}\left(\phi_{2}(t, z), a, x\right) \equiv(E \alpha)(x) \bar{T}_{1}^{\alpha}\left(\psi_{2}(t, z), a, x\right)
$$

for some primitive recursive $\phi_{2}$ and $\psi_{2}$. So it will suffice to take $\tau(y)$ $=2^{\phi_{2}\left(t,\left(y_{0}\right)\right.} \cdot 3 \psi_{2}\left(t,(y)_{0}\right)$. CASE $3: y=3 \cdot 5^{z}$ and $y \in O$. Similarly

$$
\begin{aligned}
H_{y}(a) & \equiv(\alpha)(E x) T_{1}^{\alpha}\left(\left(\tau\left(z_{(a)_{1}}\right)\right)_{0},(a)_{0}, x\right) \equiv(E \alpha)(x) \bar{T}_{1}^{\alpha}\left(\left(\tau\left(z_{(a)_{1}}\right)\right)_{1},(a)_{0}, x\right) \\
& \equiv(\alpha)(E x) T_{1}^{\alpha}\left(\phi_{3}(t, z), a, x\right) \equiv(E \alpha)(x) \bar{T}_{1}^{\alpha}\left(\psi_{3}(t, z), a, x\right) .
\end{aligned}
$$

8.4 Corollary 1. Each predicate $H_{y}^{Q}(a)$, where $Q$ is expressible in both $k+1$-quantifier forms and $y \in O^{Q}$, is expressible in both $k+1$-quantifier forms (with index $\tau_{k}(e, y)$ where $\tau_{k}$ is primitive recursive and $e$ is an index of $Q$ ). 
Proof. Take e.g. $k=0$. By the theorem, $H_{y}^{Q}(a) \equiv(\alpha)(E x) T_{1}^{Q, \alpha}\left(\left(\tau^{1}(y)\right)_{0}, a, x\right)$ $\equiv(E \alpha)(x) \bar{T}_{1}^{Q, \alpha}\left(\left(\tau^{1}(y)\right)_{1}, a, x\right)$ where $Q(x) \equiv(\alpha)(E x) T_{1}^{\alpha}\left((e)_{0}, a, x\right) \equiv(E \alpha)(x)$ $\bar{T}_{1}^{\alpha}\left((e)_{1}, a, x\right)$. Thence similarly to the last steps in the proofs of Theorems 6 and 9 Case $2, H_{y}^{Q}(a) \equiv(\alpha)(E x) T_{1}^{\alpha}\left(\phi_{0}(e, y), a, x\right) \equiv(E \alpha)(x) \bar{T}_{1}^{\alpha}\left(\psi_{0}(e, y), a, x\right)$ for some primitive recursive $\phi_{0}$ and $\psi_{0}$. Let $\tau_{0}(e, y)=2^{\phi_{0}(e, y)} \cdot 3^{\psi_{0}(e, y)}$.

CoROllaRy 2. Each predicate $H_{v}^{Q}(a)$, where $Q$ is a $k$-quantifier predicate and $y \in O^{Q}$, is expressible in both $k+1$-quantifier forms.

8.5 Corollary 3. The predicate $(\alpha)(E x) T_{1}^{\alpha}(a, a, x)$ is of degree $>0_{(y)}$ for every $y \in O$.

Proof. The degree $\mathbf{0}_{(y)}$ is possessed by the predicate $H_{y}$, which by the theorem is expressible in both 1-quantifier forms, a fortiori by the proof of Theorem 4 is recursive in $(\alpha)(E x) T_{1}^{\alpha}(a, a, x)$. But were $(\alpha)(E x) T_{1}^{\alpha}(a, a, x)$ recursive (and hence arithmetical) in $H_{y}$, by Theorem 5 it would be expressible in both 1-quantifier forms, contradicting the proof of Theorem 3.

8.6 By Theorem 9 Corollary 2 and Theorem 5 , the predicates $P(a)$ expressible in both $k+1$-quantifier forms include those recursive in $H_{v}^{Q}$ for $y \in O^{Q}$ and $Q$ a $k$-quantifier predicate (and for $k=0$, using Theorem 9 itself, those recursive in $H_{y}$ for $\left.y \in O\right)$. This suggests the conjecture that these classes of predicates are identical. The $k=0$ case of this conjecture will be confirmed in $[10]$.

9. Mostowski's extension of the arithmetical hierarchy. 9.1 In 9.4 and 9.5 we shall give a proof of the equivalence of Mostowski's extension [16, $\S 1]$ of the arithmetical hierarchy to the extension afforded by the predicates $H_{y}$ as defined for $y \in O$ in $6.2\left({ }^{19}\right)$. It is necessary to read between the lines a little in Mostowski's brief account to reach a suitable formulation of his extension, as follows $\left({ }^{23}\right)$.

(23) (a) He passes over without comment (p. 245) the possibility that different effectively defined sequences $\beta_{n}$ convergent to $\alpha$ may lead to different classes $P_{\alpha}^{(k)}$ and $Q_{\alpha}^{(k)}$; in any case, the normal universal functions $F_{\alpha}^{(k)}$ are different, besides which the notion of effectiveness when made precise, e.g. via recursiveness, applies to a system of notations for ordinals.

(b) On p. $243 F_{1}^{(k)}(m)$ should be a set of $k$-tuples based on an effective enumeration.

(c) Likewise "starting with any system of normal functions universal for the classes $P_{1}^{(h)}(h=1,2,3, \cdots)$ we obtain the same classes $P_{\alpha}^{(k)}$ and $Q_{\alpha}^{(k)}$ for any $\alpha<\omega_{1}$ " (p. 246), if the system is effective in $h$. (To give a counterexample without this effectiveness, let $F_{1}^{(h)}$ be a given system, and define another $E_{1}^{(h)}$ thus, using an arbitrary set $B$ of natural numbers: $\left(a_{1}, \cdots, a_{h}\right) \in E_{1}^{(h)}(m) \equiv a_{1}=h-1 \& a_{1} \in B$ if $m=0$ and $h$ is odd, $\equiv a_{1} \neq h-1 \bigvee a_{1} \in B$ if $m=0$ and $h$ is even, $\equiv\left(a_{1}, \cdots, a_{h}\right) \in F_{1}^{(h)}(m-1)$ if $m>0$. Now $a \in E_{n}^{(1)}(0) \equiv a=n-1 \& a \in B$. So $B=\sum_{i} E_{i+1}^{(1)}(0)=\sum_{(i, j)} \in A E_{i}^{(1)}(j)$ for $A=(\hat{i}, \hat{j})[i \neq 0 \& j=0]$; i.e. starting with $E_{1}^{(h)}, B$ would belong to $P_{\omega}^{(1)}$.)

(d) His classification at limit ordinals $\alpha$ gives what we get at $\alpha+1$. Of course this is no error, but a circumstance to be taken into account in matching the two extensions. (He puts $P_{0}^{(k)}=Q_{0}^{(k)}=P_{1}^{(k)} \cdot Q_{1}^{(k)}$, but has no ordinal left as index for $P_{\alpha}^{(k)} \cdot Q_{\alpha}^{(k)}$ for $\alpha$ a limit ordinal.)

In view of $(\mathrm{d})$, we increase by one the indices of $P_{\alpha}^{(k)}$ and $Q_{\alpha}^{(k)}$ for transfinite $\alpha$, and decrease by one the indices of $F_{\alpha}^{(k)}$ for finite $\alpha$, after which in view of (a) we replace all ordinals $\alpha$ as in- 
We define a function $F_{y}^{k}$ from natural numbers to sets of $k$-tuples of natural numbers, for every $y \in O$ and positive integer $k$, thus: $\left(a_{1}, \cdots, a_{k}\right)$ $\in F_{1}^{\boldsymbol{k}}(m) \equiv(E x) T_{k}\left(m, a_{1}, \cdots, a_{k}, x\right)$. If $2^{z} \in O$ and $z \neq 0,\left(a_{1}, \cdots, a_{k}\right) \in F_{2^{z}}^{\boldsymbol{k}}(m)$ $\equiv(E x)\left[\left(a_{1}, \cdots, \quad a_{k}, x\right) \notin F_{z}^{k+1}(m)\right]$. If $3 \cdot 5^{z} \in O, \quad\left(a_{1}, \cdots, a_{k}\right) \in F_{3 \cdot b^{z}}^{k}(m)$ $\equiv(E i)(E j)\left[(i, j) \in F_{z_{n_{1}(m)}}^{2}\left(s_{2}(m)\right) \quad \& \quad\left(a_{1}, \cdots, \quad a_{k}\right) \in F_{z_{i}}^{k}(j)\right]$ where $m+1$ $=2^{s_{1}(m)}\left(2 s_{2}(m)+1\right)\left({ }^{24}\right)$.

We define classes $P_{y}^{k}$ and $Q_{y}^{k}$ of sets of $k$-tuples of natural numbers, for every $y \in O$ and positive integer $k$, thus: Let $y^{*}=2^{y}$, so $\left|y^{*}\right|=|y|+1$. $P_{1^{*}}^{k}$ shall consist of the sets $A$ of $k$-tuples such that, for some primitive recursive predicate $R,\left(a_{1}, \cdots, a_{k}\right) \in A \equiv(E x) R\left(a_{1}, \cdots, a_{k}, x\right)$. If $2^{z} \in O$ and $z \neq 0$, $P_{\left(2^{2}\right)^{*}}^{k}=\widehat{A}\left\{\right.$ for some $B, B \in P_{z^{*}}^{k+1}$ and $\left(a_{1}\right) \cdots\left(a_{k}\right)\left\{\left(a_{1}, \cdots, a_{k}\right) \in A \equiv(E x)\right.$ $\left.\left.\left[\left(a_{1}, \cdots, a_{k}, x\right) \notin B\right]\right\}\right\}$. If $3 \cdot 5^{z} \in O, P_{\left(3 \cdot 5^{*}\right)^{*}}^{k}=\widehat{A}\left\{\right.$ for some $B$ and $n, B \in P_{z_{n}^{*}}^{2}$ and $\left(a_{1}\right) \cdots\left(a_{k}\right)\left\{\left(a_{1}, \cdots, a_{k}\right) \in A \equiv(E i)(E j)\left[(i, j) \in B \quad \&\left(a_{1}, \cdots, a_{k}\right)\right.\right.$ $\left.\left.\left.\in F_{z_{i}}^{k}(j)\right]\right\}\right\}$. If $y \in O, Q_{y^{*}}^{k}$ shall be the class of the complements of the sets belonging to $P_{y^{*}}^{k}$. If $y=1$, or $y=3 \cdot 5^{z}$ and $y \in O, P_{y}^{k}=Q_{y}^{k}=P_{v^{*}}^{k} \cdot Q_{v^{*}}^{k}$.

Now it is a theorem that, if $y \in O, P_{y^{*}}^{k}=\widehat{A}(E m)\left[A=F_{y}^{k}(m)\right]$; and also that, if $y \in O,\left(\hat{a}_{1}, \cdots, \hat{a}_{k}, \widehat{m}\right)\left[\left(a_{1}, \cdots, a_{k}\right) \in F_{y}^{\boldsymbol{k}}(m)\right] \in P_{y^{*}}^{k+1}\left({ }^{25}\right)$.

9.2 Let $\left[a_{1}, \cdots, a_{k}\right]=p_{0}^{a_{1}+1} \cdots \cdots p_{k-1}^{a_{k}+1}$, and $H_{v^{*}}^{k}\left(m, a_{1}, \cdots, a_{k}\right)$ $\equiv H_{v^{*}}\left(S_{1}^{1,1}\left(m,\left[a_{1}, \cdots, a_{k}\right]\right)\right)$.

The equivalence of Mostowski's and the present extensions of the arithmetical hierarchy will be established by showing that his $\left(a_{1}, \cdots, a_{k}\right) \in F_{y}^{k}(m)$ comes from our $H_{y^{*}}(a)$ by substitution of a recursive function (Lemma 16), and vice versa (Lemmas 17 and 18). The $\left(a_{1}, \cdots, a_{k}\right) \in F_{y}^{k}(m)$ are "enumerating predicates" (cf. Theorem 2 or 8.2 above, or IM Theorem IV p. 281), while the $H_{v^{*}}(a)$ are "complete predicates" (cf. Theorem 4 above, or IM p. 343 Example 2, or $[11,1.4])$. However the $H_{y^{*}}^{k}\left(m, a_{1}, \cdots, a_{k}\right)$ are "enum-

dices by numbers $y$ which $\in O$ in the system $S_{3}$ of notation for ordinals. For brevity, in this connection we write $y^{*}$ for $2^{v}$ (so $\left|y^{*}\right|=|y|+1$ ). Thus his $P_{1}^{(k)}$ and $F_{1}^{(k)}$ becomes $P_{10}^{k}$ and $F_{0 o}^{k}$ $\left(0_{o}=1,1_{o}=1^{*}\right)$, his $P_{\omega}^{(k)}$ and $F_{\omega}^{(k)}$ become $P_{w^{*}}^{k}$ and $F_{w}^{k}$ where $w$ is a suitable notation for $\omega$, and $F_{\nu}^{k}$ is a normal universal function for $P_{y^{k}}^{k}\left(\right.$ not for $\left.P_{\nu}^{k}\right)$.

We avoid the difficulty under (b) and (c) by using as the $F_{1}^{k}$ a particular system of normal universal functions for the $P_{1^{*}}^{k}$ which is already in the literature (IM Theorem IV p. 281). Sufficient effectiveness properties for any other system are then that it be transformable into the particular one, and vice versa, by substitution for $m$ of a recursive function of $k$ and $m$.

(Besides, we avoid several minor discrepancies: in his p. 245 line 6 for $n=0$; in p. 245 line 8 for $m$ odd and p. $244(9)$ for $(0, j) \in A$, since he has no $F_{0}^{(k)}$; and in p. 240 lines 5-6 "one-to-one" for finite classes.)

(24) Lemma 16 with this proof using 1 instead of $z_{0}$ in Case 3 (which incidentally makes the $\beta$ become primitive recursive), and inversely a modified Lemma 16 with Lemma 17 , show that Mostowski's definition of $F_{3 \cdot b^{z}}^{k}$ (as reformulated in 9.1) could have been simplified replacing $z_{s_{1}(m)}$ by 1 and $s_{2}(m)$ by $m$; or in terms of his text, on p. 245 line $8 s_{1}(m)$ and $s_{2}(m)$ could have been simply 1 and $m$.

${ }^{25}$ ) The former states the "universality", the latter the "normality", of $F_{y}^{k}$ for $P_{y}^{k}$. The universality is easily proved now; the normality will follow thence by 9.6. Putting $\left(a_{1}, \cdots, a_{k}\right)$ $\in G_{y}^{k}(m) \equiv\left(a_{1}, \cdots, a_{k}\right) \notin F_{y}^{k}(m), G_{y}^{k}$ is universal and normal for $Q_{y}^{k}$. 
erating predicates", as the next lemma shows. We effect the transition between $F_{\nu}^{\lambda}$ and $H_{\nu^{*}}$ via $H_{\nu^{*}}^{k}$

The lemma applies to a family of predicates $(E x) R_{k}^{A}\left(b_{1}, \cdots, b_{n}, a_{1}, \cdots\right.$, $\left.a_{k}, x\right)$ with $n$ fixed and $k$ variable, when the dependence on $k, a_{1}, \cdots, a_{k}$ can be reduced recursively to dependence on the number $\left[a_{1}, \cdots, a_{k}\right]$. This restriction gives the necessary effectiveness in $k$. Our applications of the lemma will be to predicates formed from $H_{u^{*}}^{\boldsymbol{k}}\left(m, a_{1}, \cdots, a_{k}\right)$ by operations in which $a_{1}, \cdots, a_{k}$ are held fixed as parameters, and then the restriction will be met in consequence of the definition of $H_{u^{*}}^{k}$; and to predicates formed similarly from $T_{k}\left(m, a_{1}, \cdots, a_{k}, x\right)$, and then the restriction will be met in consequences of IM p. 287 (22) with $u=0$, since $2^{k} \cdot p_{p_{1}}^{a_{1}+1} \cdots \cdots p_{k}^{a_{k}+1}$ can be expressed in terms of $\left[a_{1}, \cdots, a_{k}\right]$ using IM \#\#19, 20 p. 230.

Lemma 13. Given a predicate $R^{A}\left(b_{1}, \cdots, b_{n}, a, x\right)$ partial recursive uniformly in $A(a)$, there are primitive recursive functions $\phi$ and $\psi$ such that, if $y \in O$, then

$$
\begin{gathered}
(E x) R_{y}^{H}\left(b_{1}, \cdots, b_{n},\left[a_{1}, \cdots, a_{k}\right], x\right) \equiv B_{y^{*}}^{k}\left(\phi\left(b_{1}, \cdots, b_{n}\right), a_{1}, \cdots, a_{k}\right), \\
(x) R_{y}^{H}\left(b_{1}, \cdots, b_{n},\left[a_{1}, \cdots, a_{k}\right], x\right) \equiv \bar{H}_{y^{*}}^{k}\left(\psi\left(b_{1}, \cdots, b_{n}\right), a_{1}, \cdots, a_{k}\right)
\end{gathered}
$$

for values of $y, b_{1}, \cdots, b_{n}, a_{1}, \cdots, a_{k}$ which make $\lambda x R^{H_{\nu}}\left(b_{1}, \cdots, b_{n}\right.$, $\left.\left[a_{1}, \cdots, a_{k}\right], x\right)$ completely defined. (Similarly for $n=0$.)

Proof. Let $e$ be a uniform Gödel number of $\lambda b_{1} \cdots b_{n}$ at $\mu x R^{A}\left(b_{1}, \cdots, b_{n}\right.$, $a, x)$ from $A$, and let $\phi\left(b_{1}, \cdots, b_{n}\right)=S_{2}^{n, 1}\left(e, b_{1}, \cdots, b_{n}\right)$.

9.3 LEMMA 14. For each $n \geqq 0$, there is a primitive recursive function $R_{n}(z, k)$ such that, if $e$ is a Gödel number of $\lambda b_{1} \cdots b_{n} a \phi\left(b_{1}, \cdots, b_{n}, a\right)$, then, for each $k, R_{n}(e, k)$ is a Gödel number of $\lambda b_{1} \cdots b_{n} a_{1} \cdots a_{k} \phi\left(b_{1}, \cdots, b_{n}\right.$, $\left[a_{1}, \cdots, a_{k}\right]$ ). (More generally, there are $R_{n}^{m_{1}, \cdots, m_{l}}$ analogously to $S_{n}^{m, m_{1}, \cdots, m_{l}}$ IM Theorem XXIII p. 342.)

Proof. Writing $\phi\left(b_{1}, \cdots, b_{n}, a_{1}, \cdots, a_{k}\right)$ for $\phi\left(b_{1}, \cdots, b_{n},\left[a_{1}, \cdots, a_{k}\right]\right)$, the new function $\phi$ satisfies the equations

$$
\left\{\begin{aligned}
\phi\left(b_{1}, \cdots, b_{n}, a\right) & \simeq \Phi_{n+1}\left(e, b_{1}, \cdots, b_{n}, a\right) \\
\chi(a, b) & \simeq 2^{a+1} * b \\
\phi\left(b_{1}, \cdots, b_{n}, a_{1}, \cdots, a_{k}\right) & \simeq \phi\left(b_{1}, \cdots, b_{n}, \chi\left(a_{1}, \chi\left(a_{2}, \cdots \chi\left(a_{k}, 1\right) \cdots\right)\right)\right) .
\end{aligned}\right.
$$

The proof is completed from (24) similarly to that of IM Theorem XXIII from (68) (cf. the erratum in our bibliography [8]); the formal variables used in translating $b_{1}, \cdots, b_{n}, a_{1}, a_{2}, \cdots$ can be the variables in the order of their generation under IM Df2 pp. 277, 252.

Lemma 15. The function $S_{n}^{m}\left(z, y_{1}, \cdots, y_{m}\right)$ (IM Theorem XXIII) can be chosen so that, for each $m, \lambda n z y_{1} \cdots y_{m} S_{n}^{m}\left(z, y_{1}, \cdots, y_{m}\right)$ is primitive recursive. (Similarly for $S_{n}^{m, m_{1}}, \cdots, m_{l}$.) 
Proof. Using IM p. 287 (22), IM (68) can be rewritten

$$
\left\{\begin{aligned}
\Phi(z, a) & \simeq U(\mu y T(z, a, y)), \\
\phi\left(x_{1}, \cdots, x_{n}\right) & \simeq \Phi\left(e, 2^{m+n} \cdot p_{1}^{y_{1}+1} \cdots \cdot p_{m}^{y_{m}+1} \cdot p_{m+1}^{x_{1+1}} \cdots p_{m+n}^{x_{n}+1}\right) .
\end{aligned}\right.
$$

9.4 Lemma 16. There is a primitive recursive function $\alpha(y, m)$ such that, if $y \in O$, then

$$
\left(a_{1}, \cdots, a_{k}\right) \in F_{y}^{k}(m) \equiv H_{y^{*}}^{k}\left(\alpha(y, m), a_{1}, \cdots, a_{k}\right) .
$$

Proof, by induction on $y$ (cf. the proofs of Lemmas 3 and 4 in 6.3). Case 1: $y=1$. Using successively the def. of $F_{1}^{k}$, IM (22) (cf. 9.2), and Lemma 13, $\left(a_{1}, \cdots, a_{k}\right) \in F_{1}^{k}(m) \equiv H_{1^{*}}^{k}\left(\phi_{1}(m), a_{1}, \cdots, a_{k}\right)$ for a primitive recursive $\phi_{1}$. CASE 2: $y=2^{z}$ and $z \in O$. Using successively the def. of $F_{y}^{k}$, the hyp. ind., and the def. of $H_{2^{*}}^{k+1}$, then replacing $\alpha$ by $\{a\}$ and $\left[a_{1}, \cdots, a_{k}, x\right]$ by $\left[a_{1}, \cdots, a_{k}\right] * 2^{x+1}$, and finally applying Lemma $13,\left(a_{1}, \cdots, a_{k}\right) \in F_{y}^{\boldsymbol{k}}(m)$ $\equiv H_{v^{*}}^{k}\left(\phi_{2}(a, z, m), a_{1}, \cdots, a_{k}\right)$ for a primitive recursive $\phi_{2}$, when $a$ is a Gödel number of $\alpha$. CASE 3: $y=3 \cdot 5^{z}$ and $y \in O$. Using successively the def. of $F_{\boldsymbol{y}}^{\boldsymbol{k}}$, the hyp. ind., and the defs. of $H_{z_{n}}^{k}, H_{z_{n}}$, and $H_{y}$, then advancing $(E x)$, contracting $(E i)(E j)\left(E x_{1}\right)\left(E x_{2}\right)$, replacing $\alpha$ by $\{a\}$, and applying Lemma 13, $\left(a_{1}, \cdots, a_{k}\right) \in F_{\nu}^{k}(m) \equiv H_{\nu^{*}}^{k}\left(\phi_{3}(a, z, m), a_{1}, \cdots, a_{k}\right)$ for a primitive recursive $\phi_{3}$, when $a$ is a Gödel number of $\alpha$.

9.5 Lemma 17. There is a partial recursive function $\beta(k, y, m)$ such that, if $y \in O$, then

$$
H_{y^{*}}^{k}\left(m, a_{1}, \cdots, a_{k}\right) \equiv\left(a_{1}, \cdots, a_{k}\right) \in F_{y}^{k}(\beta(k, y, m)) .
$$

Proof, by induction on $y$. CASE 1: $y=1$. Using the defs. of $H_{1^{*}}^{k}, H_{1^{*}}$ and $H_{1}$, then Lemmas 14 and 15 (cf. IM p. 343 Example 3), and finally the def. of $F_{1}^{k}, H_{1}^{k}\left(m, a_{1}, \cdots, a_{k}\right) \equiv\left(a_{1}, \cdots, a_{k}\right) \in F_{1}^{k}\left(S_{k}^{1}\left(R_{1}(e, k), m\right)\right)$ when $e$ is a Gödel number of $\lambda \operatorname{ma} \mu x T_{1}^{\lambda a a=a}\left(S_{1}^{1,1}(m, a), S_{1}^{1,1}(m, a), x\right)$. CASE 2: $y=2^{z}$ and $z \in O$. Then $H_{y}(a) \equiv(E y) T_{1}^{H_{z}}(a, a, y)$, so by IM Theorem XI* pp. 293, 295 $T_{1}^{H_{y}}(a, a, x)$ is expressible in both 2 -quantifier forms relative to $H_{z}$, in particular with the prefix $(E w)(y)$. Using the defs. of $H_{v^{*}}^{\boldsymbol{k}}$ and $H_{v^{*}}$, and the above fact, and contracting $(E x)(E w), H_{y^{*}}^{\boldsymbol{k}}\left(m, a_{1}, \cdots, a_{k}\right) \equiv(E x)(y) R^{\boldsymbol{H}_{\boldsymbol{z}}}$ $\left(m,\left[a_{1}, \cdots, a_{k}, x\right], y\right)$ where $R^{A}$ is recursive uniformly in $A$. By Lemma 13, $(y) R^{H_{z}}\left(m,\left[a_{1}, \cdots, a_{k}, x\right], y\right) \equiv \bar{H}_{y}^{k+1}\left(\psi(m), a_{1}, \cdots, a_{k}, x\right)$ for a primitive recursive $\psi$. So using the hyp. ind. and the def. of $F_{\nu}^{k}, H_{\nu^{*}}^{k}\left(m, a_{1}, \cdots, a_{k}\right)$ $\equiv\left(a_{1}, \cdots, a_{k}\right) \in F_{y}^{k}(\beta(k+1, z, \psi(m)))$. CASE 3: $y=3 \cdot 5^{z}$ and $y \in O$. By the def. of $T_{1}^{A}$ (IM p. 291), $T_{1}^{H_{y}}(a, a, x)$ depends on only the first $x$ values of $H_{y}$. But for $c<x, H_{y}(c) \equiv H_{z(c)_{1}}\left((c)_{0}\right) \equiv H_{z_{x}}\left(\rho\left(z_{(c)_{1}}, z_{x},(c)_{0}\right)\right)$, by Lemma 3. Now $H_{y^{*}}^{k}\left(m, a_{1}, \cdots, a_{k}\right) \equiv(E x) H_{z_{x}^{*}}^{k}\left(\phi(z, m, x), a_{1}, \cdots, a_{k}\right)$ [by the defs. of $H_{y^{*}}^{k}$ and $H_{\nu^{*}}$, the above facts, and Lemma 13, for a primitive recursive $\phi$ ] $\equiv(E x)\left\{\left(a_{1}, \cdots, a_{k}\right) \in F_{z_{x}}^{k}\left(\beta\left(k, z_{x}, \phi(z, m, x)\right)\right)\right\} \quad[$ hyp. ind. $] \equiv(E x)(E i)(E j)$ $\left\{i=x \quad \& \quad j=\beta\left(k, \quad z_{x}, \phi(z, \quad m, x)\right) \&\left(a_{1}, \cdots, \quad a_{k}\right) \in F_{z_{i}}^{k}(j)\right\} \equiv(E i)(E j)$ 
$\left\{(E x)\left[i=x \& j=\beta\left(k, z_{x}, \phi(z, m, x)\right)\right] \&\left(a_{1}, \cdots, a_{k}\right) \in F_{z_{i}}^{k}(j)\right\} \equiv(E i)(E j)$ $\left\{(i, j) \in F_{z_{0}}^{2}\left(\beta\left(2, z_{0}, \phi(b, k, z, m)\right)\right) \&\left(a_{1}, \cdots, a_{k}\right) \in F_{z_{i}}^{k}(j)\right\}$ [by Lemma 13, for $b$ a Gödel number of $\beta$, and a primitive recursive $\phi$; and the hyp. ind.] $\equiv\left(a_{1}, \cdots, a_{k}\right) \in F_{\boldsymbol{v}}^{\boldsymbol{k}}\left(2 \beta\left(2, z_{0}, \phi(b, k, z, m)\right)\right)$ [by the def. of $\left.F_{\nu^{*}}^{k}\right]\left({ }^{25}\right)$.

LEMMA 18. There is a number $f$ such that, if $y \in O$, then

$$
H_{y^{*}}(a) \equiv H_{y^{*}}^{1}(f, a) \text {. }
$$

Proof. Use the def. of $H_{y^{*}}$ and Lemma 13 with $a=([a])_{0}-1$.

9.6(24) Using Lemma 16, the defs. of $H_{y^{*}}^{k}$ and $H_{y^{*}}$, and Lemmas 13 and 17, $\left(a_{1}, \cdots, a_{k}\right) \in F_{y}^{\boldsymbol{k}}(m) \equiv\left(a_{1}, \cdots, a_{k}, m\right) \in F_{y}^{\boldsymbol{k}+1}(\beta(k+1, y, \phi(y)))$ for a primitive recursive $\phi$.

9.7 The definitions of $F_{\nu}^{k}, P_{\nu}^{\boldsymbol{k}}, Q_{\nu}^{\boldsymbol{k}}, H_{\nu^{*}}^{\boldsymbol{k}}$ and the theory can be relativized to say a 1 -place predicate $Q$. Besides carrying the superscript "Q" on $O,<0$, $z_{n}\left(=\{z\}\left(n_{0}\right)\right), F_{y}^{k}, P_{y}^{k}, Q_{y}^{k}, H_{y}, H_{y^{*}}^{k}$, etc., in 9.1 we use $T_{k}^{\ell}$ instead of $T_{k}$, and an $R^{Q}$ primitive recursive in $Q$ instead of the primitive recursive $R$. In 9.2, in the definition of $H_{v^{*}}^{k}$, we use $S_{1}^{1,1,1}$ instead of $S_{1}^{1,1}$, and in Lemma 13 a predicate $R^{Q, A}$ partial recursive uniformly in $Q, A$ instead of the $R^{A}$. In 9.4, $\alpha$ becomes a primitive recursive $\alpha^{1}$. In $9.5, \beta$ becomes a $\beta^{Q}$ partial recursive uniformly in $Q$, and $f$ becomes $f^{1}$. Lemmas $13,16-18$ thus relativized we cite as Lemmas $13^{*}, 16^{*}-18^{*}$.

9.8(16) By the definitions of $F_{y}^{Q, k}(9.1,9.7)$ and $n_{0}(6.1)$,

$$
\begin{aligned}
a & \in F_{(2 n)_{0}}^{Q, 1}(m) \\
& \equiv\left(E x_{1}\right)\left(y_{1}\right) \cdots\left(E x_{n}\right)\left(y_{n}\right)\left(E x_{n+1}\right) T_{2 n+1}^{Q}\left(m, a, x_{1}, y_{1}, \cdots, x_{n}, y_{n}, x_{n+1}\right), \\
a & \in F_{(2 n+1)_{o}}^{Q, 1}(m) \\
& \equiv\left(E x_{1}\right)\left(y_{1}\right) \cdots\left(E x_{n+1}\right)\left(y_{n+1}\right) \bar{T}_{2 n+2}^{Q}\left(m, a, x_{1}, y_{1}, \cdots, x_{n+1}, y_{n+1}\right) .
\end{aligned}
$$

Hence for the $N_{i}^{Q}(a)$ in the proof of Theorem 6 ,

$$
\begin{aligned}
& N_{2 n+1}^{Q}(a) \equiv a \in F_{(2 n)_{o}}^{Q, 1}(a), \\
& N_{2 n+2}^{Q}(a) \equiv a \in F_{(2 n+1)_{o}}^{Q, 1}(a) .
\end{aligned}
$$

Using in succession (31), Lemma $16^{*}$, the definition of $H_{(2 n+1) o}^{Q, 1}$, Theorem 9, and Lemma $12, N_{2 n+1}^{Q}(a) \equiv(\alpha)(E x) T_{1}^{Q, \alpha}\left(\phi_{2}(n), a, x\right) \equiv(E \alpha)(x) \bar{T}_{1}^{Q, \alpha}\left(\psi_{2}(n), a, x\right)$ for some primitive recursive $\phi_{2}$ and $\psi_{2}$. A similar formula for $N_{2 n+2}^{Q}(a)$ is obtained likewise by starting from (32), and for $N_{0}^{Q}(a)$ simply by Theorem 2 . Then $\eta(i)$ and $\theta(i)$ are defined by cases $(i=0, i=2 n+1, i=2 n+2)$.

9.9 Lemma 19 is used in 9.10 in proving a result alluded to in [11, Footnote 29, " $L_{k}^{A}$ IN $N_{k}^{A}$ "].

Lemma 19. There is a primitive recursive function $\zeta(i, m, a)$ such that 


$$
\begin{aligned}
(E y) T_{i+1}^{Q}\left(m, a, x_{1}, \cdots\right. & \left., x_{i}, y\right) \\
& \equiv(E y) T_{i+1}^{Q}\left(\zeta(i, m, a), \zeta(i, m, a), x_{1}, \cdots, x_{i}, y\right) .
\end{aligned}
$$

Proof. Using IM (22) pp. 287, 292, $T_{i+1}^{Q}\left(m, a, x_{1}, \cdots, x_{i}, y\right) \equiv R^{Q}(m, a$, $\left.\left[x_{1}, \cdots, x_{i}\right], y\right)$ with an $R^{Q}$ primitive recursive uniformly in $Q$. So using Lemma 14 with IM p. 343 Example 2, and Lemma 15, it suffices to take $\zeta(i, m, a)=S_{i+1}^{2,1}\left(R_{3}^{1}(e, i), m, a\right)$ for $e$ a uniform Gödel number from $Q$ of $\lambda m a t v \mu y R^{Q}(m, a, v, y)$.

9.10 Using (29) for $i$ even and (30) for $i$ odd, with (33),

$$
a \in F_{i_{o}}^{Q, 1}(m) \equiv \zeta(i, m, a) \in F_{i_{o}}^{Q, 1}(\zeta(i, m, a)) .
$$

Using Lemmas $10,18^{*}$ and $17^{*}\left({ }^{26}\right)$, with (34), and (31) or (32),

$$
\begin{aligned}
& \dot{H}_{(2 n+1)_{o}}^{Q}(a) \equiv N_{2 n+1}^{Q}\left(\zeta\left(2 n, \beta^{Q}\left(1,(2 n)_{o}, f^{1}\right), \mu^{Q}\left((2 n+1)_{o}, a\right)\right)\right), \\
& \dot{H}_{(2 n+2)_{o}}^{Q}(a) \equiv \bar{N}_{2 n+2}^{Q}\left(\zeta\left(2 n+1, \beta^{Q}\left(1,(2 n+1)_{o}, f^{1}\right), \mu^{Q}\left((2 n+2)_{o}, a\right)\right)\right) .
\end{aligned}
$$

The result " $L_{\boldsymbol{k}}^{A}$ IN $N_{\boldsymbol{k}}^{A}$ ", with a primitive recursive $\epsilon$, now follows easily from $L_{i}^{Q}(a) \equiv \dot{H}_{t_{0}}^{Q}(a)$ with $\dot{H}_{0_{o}}^{Q}(a) \equiv N_{0}^{Q}(a)$, (35) and (36).

\section{BIBLIOGRAPHY}

1. A. Church, $A$ set of postulates for the foundation of logic, Ann. of Math. (2) vol. 33 (1932) pp. 346-366.

2. M. Davis, On the theory of recursive unsolvability, $\mathrm{Ph} . \mathrm{D}$. thesis (typewritten), Princeton University, 1950.

3. - Relatively recursive functions and the extended Kleene hierarchy, Proceedings of the International Congress of Mathematicians (Cambridge, Mass., U.S.A., Aug. 30-Sept. 6, 1950), 1952, vol. 1, p. 723. 155.

4. S. C. Kleene, On notation for ordinal numbers, J. Symbolic Logic vol. 3 (1938) pp. 150

5. - Recursive predicates and quantifiers, Trans. Amer. Math. Soc. vol. 53 (1943) pp. 41-73. Cf. the note under [6].

6. - On the forms of the predicates in the theory of constructive ordinals, Amer. J. Math. vol. 66 (1944) pp. 41-58. For an error in this and [5], cf. the bibliography of [7] or [8], or [9] where a corrected treatment is given.

7. - Recursive functions and intuitionistic mathematics, Proceedings of the International Congress of Mathematicians (Cambridge, Mass., U.S.A., Aug. 30-Sept. 6, 1950), 1952, vol. 1, pp. 679-685. Erratum: p. 680 1. 8, after "that" insert "for $n>1$ ".

8. - Introduction to metamathematics, New York, Van Nostrand, Amsterdam, North Holland, and Groningen, Noordhoff, 1952, 10+550 pp. Erratum (1952 printing): p. 3421.7 from below, insert "[ 2 exp" after " *" and "]" at the end.

9. - On the forms of the predicates in the theory of constructive ordinals (second paper), to appear in Amer. J. Math.

10. - Hierarchies of number-theoretic predicates, Bull. Amer. Math. Soc. vol. 61 (1955) pp. 193-213.

${ }^{(26)}$ We can have $\beta^{Q}$ and $\mu^{Q}$ primitive recursive here, e.g. by omitting Case 3 for Lemmas $17^{*}$ and 10. By Lemmas 10 and $1, \dot{H}_{y}^{*}(a) \equiv H_{y}^{Q} \cdot\left(\mu^{1}(y, a)\right)$ with primitive recursive $\mu^{1(25)}$. 
11. S. C. Kleene and E. L. Post, The upper semi-lattice of degrees of recursive unsolvability, Ann. of Math. (2) vol. 59 (1954) pp. 379-407.

12. P. Lorenzen, Die Definition durch vollständige Induktion, Monatshefte für Mathematik und Physik vol. 47 (1938-1939) pp. 356-358.

13. G. Kreisel, On the interpretation of non-finitist proofs, J. Symbolic Logic vol. 16 (1951) pp. 241-267 and vol. 17 (1952) pp. 43-58.

14. - A variant to Hilbert's theory of the foundations of arithmetic, The British Journal for the Philosophy of Science vol. 4 (1953) pp. 107-129.

15. A. Mostowski, On definable sets of positive integers, Fund. Math. vol. 34 (1946) pp. 81112.

16. _ - A classification of logical systems, Studia Philosophica vol. 4 (1951) pp. 237274.

17. J. Myhill, Arithmetic with creative definitions by induction, J. Symbolic Logic vol. 18 (1953) pp. 115-118.

18. R. Péter, Über den Zusammenhang der verschiedenen Begriffe der rekursiven Funktion, Math. Ann. vol. 110 (1934) pp. 612-632.

19. E. L. Post, Recursively enumerable sets of positive integers and their decision problems, Bull. Amer. Math. Soc. vol. 50 (1944) pp. 284-316.

20. - Degrees of recursive unsolvability, Bull. Amer. Math. Soc. Abstract 54-7-269.

21. Th. Skolem, Über die Zurückführbarkeit einiger durch Rekursionen definierten Relationen auf "arithmetische", Acta litterarum ac scientiarum Regiae Universitatis Hungaricae Franccisco-Iosephinae, Sectio scientiarum mathematicarum (Szeged) vol. 8 (1936-37) pp. 73-88.

22. C. Spector, Recursive well-orderings, to appear in J. Symbolic Logic.

23. A. Tarski, Einige Betrachtungen über die Begriffe der $\omega$-Widerspruchsfreiheit und der $\omega$-Vollständigkent, Monatshefte für Mathematik und Physik vol. 40 (1933) pp. 97-112.

24. - A problem concerning the notion of definability, J. Symbolic Logic vol. 13 (1948) pp. 107-111.

25. H. Wang, Certain predicates defined by induction schemata, J. Symbolic Logic vol. 18 (1953) pp. 49-59.

The University of Wisconsin, Madison, Wis. 